\title{
Catadioptric Visual Servoing From 3-D Straight Lines
}

\author{
Hicham Hadj-Abdelkader, Youcef Mezouar, Philippe Martinet, and François Chaumette, Member, IEEE
}

\begin{abstract}
In this paper, we consider the problem of controlling a 6 DOF holonomic robot and a nonholonomic mobile robot from the projection of 3-D straight lines in the image plane of central catadioptric systems. A generic central catadioptric interaction matrix for the projection of 3-D straight lines is derived using an unifying imaging model valid for an entire class of cameras. This result is exploited to design an image-based control law that allows us to control the $6 \mathrm{DOF}$ of a robotic arm. Then, the projected lines are exploited to control a nonholonomic robot. We show that as when considering a robotic arm, the control objectives are mainly based on catadioptric image feature and that local asymptotic convergence is guaranteed. Simulation results and real experiments with a 6 DOF eye-to-hand system and a mobile robot illustrate the control strategy.
\end{abstract}

Index Terms-3-D straight lines, omnidirectional vision, visual servoing.

\section{INTRODUCTION}

$\mathbf{V}$ ISION-BASED control schemes are flexible and effective methods to control robot motions from visual data [23]. They are traditionally classified into three groups, namely position-based, image-based, and hybrid-based control [16], [23], [29]. These three schemes make assumptions on the link between the initial, current, and desired images since they require correspondences between the features extracted from the initial image with those obtained from the desired one. These measures are then tracked during the camera (and/or the object) motion. If one of these steps fails, then the task cannot be achieved. Typical cases of failure arise when matching joint image features is impossible (for example, when no joint feature belongs to initial and desired images) or when some parts of the visual features get out of the field of view during the servoing. In the later case, some methods have been investigated to resolve this deficiency based on path planning [32], [33], switching control [13], zoom adjustment [6], geometrical and

Manuscript received February 28, 2007; revised October 22, 2007. This paper was recommended for publication by Associate Editor P. Rocco and Editor L. Parker upon evaluation of the reviewers' comments. This work was supported in part by the Robotique et Entites Artificuelles-Omnidirectional vision for Robotics (ROBEA-OMNIBOT) project.

H. Hadj-Abdelkader was with the LAboratoire des Sciences et Materiaux pour l'Electronique et d'Automatique (LASMEA), Blaise Pascal University, Aubiere 63177, France. He is now with the Institut National de Recherche en Informatique et en Automatique (INRIA)-Advanced Robotics and Autonomous System (AROBAS) Project, Sophia Antipolis 06902, France (e-mail: hicham.hadj_abdelkader@sophia.inria.fr).

Y. Mezouar and P. Martinet are with the LAboratoire des Sciences et Materiaux pour l'Electronique et d'Automatique (LASMEA), Blaise Pascal University, Aubiere 63177, France (e-mail: mezouar@univ-bpclermont.fr; martinet@univbpclermont.fr).

F. Chaumette is with the Institut de Recherche en Informatique et Systemes Aleatoires (IRISA)/Institut National de Recherche en Informatique et en Automatique (INRIA), Rennes 35042, France (e-mail: francois.chaumette@ irisa.fr).

Color version of one or more of the figures in this paper are available online at http://ieeexplore.ieee.org.

Digital Object Identifier 10.1109/TRO.2008.919288 topological considerations [14], [42]. However, such strategies are sometimes delicate to adapt to generic setup.

Conventional cameras thus suffer from restricted field of view. Many applications in vision-based robotics, such as mobile robot localization [8] and navigation, [46] can benefit from a panoramic field of view provided by omnidirectional cameras. In the literature, there have been several methods proposed for increasing the field of view of cameras systems [7]. One effective way is to combine mirrors with conventional imaging system. The obtained sensors are referred to as catadioptric imaging systems. The resulting imaging systems have been termed central catadioptric when a single projection center describes the world-image mapping. From a theoretical and practical point of view, a single center of projection is a desirable property for an imaging system [2]. Baker and Nayar [2] derive the entire class of catadioptric systems with a single viewpoint. Clearly, visual servoing applications can also benefit from such sensors since they naturally overcome the visibility constraint. Vision-based control of robotic arms, single mobile robots or formations of mobile robots thus appear in the literature with omnidirectional cameras (for example, [5], [9], [38], [45]).

The interaction matrix plays a central role in designing visionbased control laws. It links the variations of image observations to the camera velocity. The analytical form of the interaction matrix is available for several image features (points, circles, lines, moments, etc.) in the case of conventional cameras [11], [16]. Barreto et al. [5] determined the central catadioptric interaction matrix for a set of image points. This paper is mainly concerned with the use of projected lines extracted from central catadioptric images as input to a visual servoing control loop. When dealing with real environments (indoor or urban) or industrial workpieces, straight line features are natural choices. Even so, most of the effort in visual servoing has been devoted to points [23] and only a few works have investigated the use of lines with traditional cameras [1], [16], [27], [28]. More importantly, none have explored the case of omnidirectional cameras as considered in this paper. Based on the preliminary research presented in [34] and [20], we first derive a generic analytical form of the central catadioptric interaction matrix for the image of 3-D straight lines. This can then be exploited to design control laws for positioning task of a 6 or less DOF manipulator.

Image-based visual servoing methods were originally developed for manipulators. Tsakiris et al. [43] point out that image-based visual servoing techniques can be extended to nonholonomic mobile robots by adding DOF to the hand-eye system. This paper proposes to embed the visual servoing control scheme in the task function formalism [16]. Vision-based mobile robotic tasks such as wall following or self-positioning with respect to landmarks is thus possible using this framework [24]. Without these extra DOFs, the pose of the camera 
with respect to the target cannot be stabilized involving only a state feedback [37]. However, it is possible to exploit work that aims to control a nonholonomic wheeled mobile robot moving on a plane [25] in order to track a nontimed analytical path in the image space without recovering any 3-D parameters of the path. Ma et al. [26] propose a theoretical framework to track a ground curve by approximating its projection in the image plane of a conventional camera with piecewise analytic curves with linear curvature. Usher et al. [44] propose a switching controller to regulate the pose of a vehicle using information provided by an omnidirectional camera. The problem of formation control is addressed in [45], by specifying the desired formation in the image plane of an omnidirectional camera. The global control problem is translated into separate visual servoing tasks for each follower. The authors address the problem of following a desired trajectory extracted from a prerecorded set of images of a stationary target in [12]. In that aim, the authors propose to measure the error between the current and desired configurations of the robot from homographic relationships. The control law is based on a Lyapunov analysis and allows compensating the unknown scale parameter, which naturally appears when extracting the translational part of the homography matrix. A central catadioptric camera is considered in [31] but as in [12], the method proposed in [31] exploits the epipolar geometry estimated from the projection of a set of points onto the image plane. The control scheme proposed in [31] is divided in two parts: first, the rotational error between the two configurations is compensated for, and then, the translational error is zeroed. In this paper, the rotational and translational errors are zeroed simultaneously with a single control law. We particularly focus on a suitable catadioptric image-based control strategy of a nonholonomic robot in order to follow a 3-D straight line. The first contribution is to formulate the control objectives in the catadioptric image space. The second one is to tightly couple catadioptric visual servoing and mobile robot control. Indeed, the control law is designed according to a well-suited chained system with a state vector directly expressed in the image space. It is shown that the observation vector used to control a manipulator using the task function formalism can also be exploited to design a control scheme based on the chained system formalism.

The remainder of this paper is organized as follows. In Section II, following the description of the central catadioptric camera model, the geometric and kinematic properties of lines in the image plane are studied. This is achieved using the unifying theory for central panoramic systems introduced in [19]. In Sections III and Section IV, we exploit the results presented in Section II to design an image-based control for a manipulator and a mobile robot. Simulation results and real experiments with a 6 DOF manipulator and a mobile robot illustrate the control strategies.

\section{MODELING}

In this section, we describe the projection model for central catadioptric cameras, and then, we focus on the geometric and kinematic models for projected 3-D straight lines.

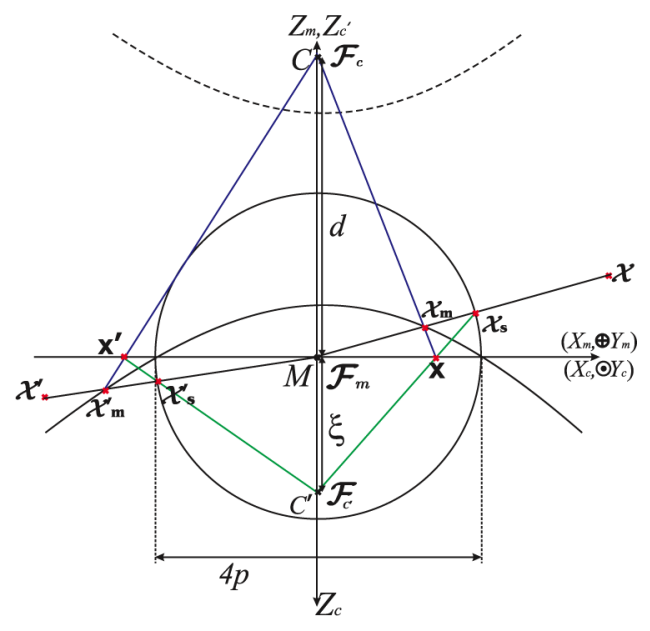

Fig. 1. Generic camera model.

TABLE I

CENTRAL CATADIOPTRIC CAMERAS

\begin{tabular}{|l|c|c|}
\hline camera & $\xi$ & $\varphi$ \\
\hline Parabolic & 1 & $1+2 p$ \\
\hline Hyperbolic & $\frac{d}{\sqrt{d^{2}+4 p^{2}}}$ & $\frac{d+2 p}{\sqrt{d^{2}+4 p^{2}}}$ \\
\hline Elliptical & $\frac{d}{\sqrt{d^{2}+4 p^{2}}}$ & $\frac{d-2 p}{\sqrt{d^{2}+4 p^{2}}}$ \\
\hline Planar & 0 & 1 \\
\hline Conventional & 0 & 1 \\
\hline
\end{tabular}

\section{A. Camera Model}

As noted previously, a single center of projection is a desirable property for an imaging system. A single center implies that all lines passing through a 3-D point and its projection in the image plane pass through a single point. Conventional perspective cameras are single view point sensors. As shown in Baker and Nayar [2], a central catadioptric system can be built by combining a hyperbolic, elliptical, or planar mirror with a perspective camera and a parabolic mirror with an orthographic camera. To simplify notations, conventional perspective cameras will be embedded in the set of central catadioptric cameras. A unifying theory for central panoramic systems is presented in [19]. According to this generic model, all central panoramic cameras can be modeled by a central projection onto a sphere followed by a central projection onto the image plane (Fig. 1). This generic model can be parametrized by the couple $(\xi, \varphi)$ defined by the mirror parameters (see Table I and [5]).

Fig. 1 clearly shows the equivalence between the direct model and the unified one in the case of a hyperbolic mirror. Let $\mathcal{F}_{c}$ and $\mathcal{F}_{m}$ be the frames attached to the conventional camera and to the mirror, respectively. In the unified model, the spherical mirror attached to the frame $\mathcal{F}_{m}$ and centered in $M$ is associated with a virtual perspective camera attached to the frame $\mathcal{F}_{c^{\prime}}$. These frames $\mathcal{F}_{m}$ and $\mathcal{F}_{c^{\prime}}$ are related by a translation of $\xi$ along the $Z$-axis. The origins $C, C^{\prime}$ and $M$ will be termed optical center, virtual optical center and principal projection center, respectively. Let $\mathcal{X}$ be a $3-\mathrm{D}$ point with coordinates $\mathbf{X}=[X, Y, Z]^{\top}$ with respect to $\mathcal{F}_{m}$. According to the generic projection model [19], $\mathcal{X}$ is projected in the image plane to a 


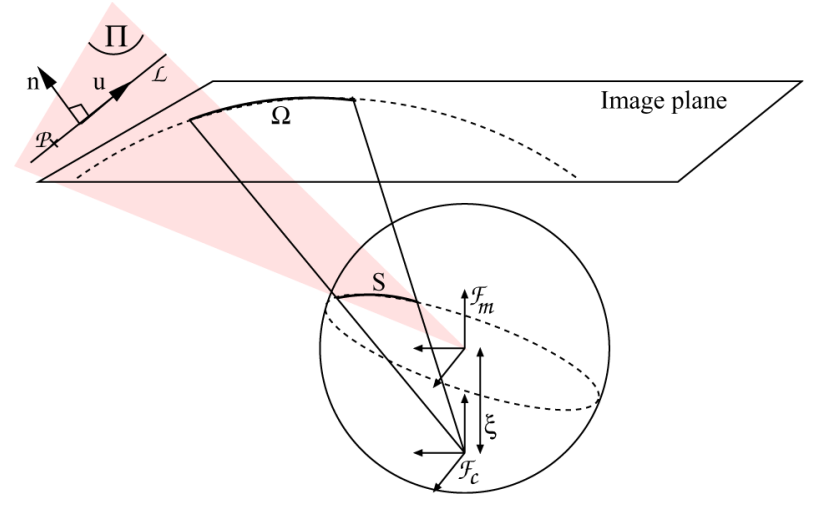

Fig. 2. Projection of line onto conic in the image plane.

point defined by the coordinates $\mathbf{x}=[x, y]^{\top}$ with

$$
\underline{\mathbf{x}}=\mathbf{K} \mathbf{f}(\mathbf{X})
$$

where $\underline{\mathbf{x}}=[x, y, 1]^{\top}$ and $\mathbf{K}$ is a plane-to-plane collineation triangular matrix and

$$
\mathbf{f}(\mathbf{X})=\left(\begin{array}{c}
\frac{X}{Z+\xi \sqrt{X^{2}+Y^{2}+Z^{2}}} \\
\frac{Y}{Z+\xi \sqrt{X^{2}+Y^{2}+Z^{2}}} \\
1
\end{array}\right) .
$$

Matrix $\mathbf{K}$ can be written $\mathbf{K}=\mathbf{K}_{\mathbf{c}} \mathbf{M}$ where the upper triangular matrix $\mathbf{K}_{\mathbf{c}}$ contains the conventional camera intrinsic parameters, and the diagonal matrix $\mathbf{M}$ contains the mirror intrinsic parameters

$$
\mathbf{M}=\left[\begin{array}{ccc}
\varphi-\xi & 0 & 0 \\
0 & -\varphi+\xi & 0 \\
0 & 0 & 1
\end{array}\right], \quad \mathbf{K}_{\mathbf{c}}=\left[\begin{array}{ccc}
\alpha_{u} & \alpha_{u v} & 0 \\
0 & \alpha_{v} & 0 \\
0 & 0 & 1
\end{array}\right]
$$

Note that for a conventional camera, $\mathcal{F}_{c^{\prime}}$ and $\mathcal{F}_{m}$ are superposed. In the sequel, we will assume without loss of generality that the matrix $\mathbf{K}$ is equal to the identity matrix; the mapping function describing central catadioptric projection is then given by $\underline{\mathbf{x}}=\mathbf{f}(\mathbf{X})$.

\section{B. Projection of Straight Lines}

In order to model the projection of lines in the image of a central imaging system, we use the Plücker coordinates of lines (Fig. 2). Let $\mathcal{P}$ be a 3-D point and $\mathbf{u}=\left[u_{x}, u_{y}, u_{z}\right]^{\top}$ a $(3 \times 1)$ vector expressed in the mirror frame and $\mathcal{L}$ the 3-D line they define. Define $\mathbf{n}=(\overrightarrow{M \mathcal{P}} \times \mathbf{u}) /(\|\overrightarrow{M \mathcal{P}} \times \mathbf{u}\|)=\left[n_{x}, n_{y}, n_{z}\right]^{\top}$ and note that this vector is independent of the point we choose on the line. Thus, the Euclidean Plücker coordinates are defined as $\mathcal{L}:\left[\begin{array}{ll}\mathbf{n}, & \mathbf{u}\end{array}\right]^{\top}$ with $\|\mathbf{n}\|=1,\|\mathbf{u}\|=1$, and $\mathbf{n}^{\top} \mathbf{u}=0$. The normal unitary vector $\mathbf{n}$ is orthogonal to the interpretation plane $\Pi$ defined by the line and the principal projection center

$$
\mathbf{X}=[X, Y, Z]^{\top} \in \Pi \Longleftrightarrow n_{x} X+n_{y} Y+n_{z} Z=0 .
$$

Let $\mathcal{S}$ be the intersection between the interpretation plane and the mirror surface. $\mathcal{S}$ represents the line projection in the mirror surface. The projection $\mathcal{S}$ of $\mathcal{L}$ in the image is then obtained using perspective mapping. It can be shown (using (1) and (2) or following [3]) that 3-D points lying on $\mathcal{L}$ are mapped into points $\mathbf{x}$ in the image which verify

$$
\underline{\mathbf{x}}^{\top} \boldsymbol{\Omega} \underline{\mathbf{x}}=0
$$

with

$$
\boldsymbol{\Omega} \propto\left(\begin{array}{ccc}
\alpha n_{x}^{2}-n_{z}^{2} \xi^{2} & \alpha n_{x} n_{y} & n_{x} n_{z} \\
\alpha n_{x} n_{y} & \alpha n_{y}^{2}-n_{z}^{2} \xi^{2} & n_{y} n_{z} \\
n_{x} n_{z} & n_{y} n_{z} & n_{z}^{2}
\end{array}\right)
$$

where $\alpha=1-\xi^{2}$. A line in space is thus mapped onto the image plane to a conic curve. The relation (3) defines a quadratic equation

$$
A_{0} x^{2}+A_{1} y^{2}+2 A_{2} x y+2 A_{3} x+2 A_{4} y+A_{5}=0
$$

where $A_{0}=\alpha n_{x}^{2}-n_{z}^{2} \xi^{2}, A_{1}=\alpha n_{y}^{2}-n_{z}^{2} \xi^{2}, A_{2}=\alpha n_{x} n_{y}$, $A_{3}=n_{x} n_{z}, A_{4}=n_{y} n_{z}$, and $A_{5}=n_{z}^{2}$ are the elements of matrix $\Omega$. Let us note that (4) is defined up to a scale factor. To obtain an unambiguous representation (4) can be normalized with one of the elements of the matrix $\Omega$ or a linear or a nonlinear combination of the elements of the matrix $\Omega$. This normalization introduces degenerate configurations that can be detected by analyzing the elements of $\Omega$. It is thus possible to adapt the normalization factor to the line configurations. This choice can be done offline if the proposed control scheme is coupled to a path planning step (as the one proposed in [21]), or alternatively, the scale factor can be chosen during the servoing process. In the sequel, the derivation of the interaction matrix is illustrated with a normalization of (4) by $A_{5}$. In this case, $A_{5}=n_{z}^{2}=0$ corresponds to a degenerate configuration where the optical axis lies on the interpretation plane, in which case, the image of the line is a straight line with equation $y=-n_{y} / n_{x} x$. Note that the interaction matrix can be obtained and exploited in a similar way with another choice for the scale factor.

Equation (4) is thus normalized using $A_{5}$

$$
B_{0} x^{2}+B_{1} y^{2}+2 \quad B_{2} x y+2 B_{3} x+2 \quad B_{4} y+1=0
$$

with $B_{i}=A_{i} / A_{5}$. More precisely

$$
\begin{array}{cc}
B_{0}=\alpha B_{3}^{2}-\xi^{2} & B_{1}=\alpha B_{4}^{2}-\xi^{2} \quad B_{2}=\alpha B_{3} B_{4} \\
B_{3}=\frac{n_{x}}{n_{z}} & B_{4}=\frac{n_{y}}{n_{z}}
\end{array}
$$

Since $B_{0}, B_{1}$, and $B_{2}$ are combination of $B_{3}$ and $B_{4}$, only two elements $B_{3}$ and $B_{4}$ are used to define the catadioptric image of straight line. As we will see in the sequel, these two elements are used to construct the observation vector that allow us to design vision-based control schemes for a 6 DOF manipulator and a nonholonomic mobile-robot. Let us note that the normal vector $\mathbf{n}$ can be computed from (6) since $\|\mathbf{n}\|=1$. We obtain

$$
\left\{\begin{array}{l}
n_{z}=\left(B_{3}^{2}+B_{4}^{2}+1\right)^{-1 / 2}=b \\
n_{x}=B_{3} b \\
n_{y}=B_{4} b
\end{array}\right.
$$


Since $\mathbf{n}^{\top} \mathbf{u}=0$, note also that $u_{z}$ can be rewritten as

$$
u_{z}=-\left(B_{3} u_{x}-B_{4} u_{y}\right) \text {. }
$$

\section{Interaction Matrix of Central Catadioptric Cameras for Conics}

Recall that the time variation $\dot{\mathbf{s}}$ of the visual features $\mathbf{s}$ can be expressed linearly with respect to the relative camera-object kinematics screw $\boldsymbol{\tau}$ (containing the instantaneous angular velocity $\boldsymbol{\omega}$ and the instantaneous linear velocity $\mathbf{v}$ of the origin of $\mathcal{F}_{m}$ expressed in the mirror frame) by

$$
\dot{\mathbf{s}}=\mathbf{L} \tau
$$

where $\mathbf{L}$ is the interaction matrix related to $\mathbf{s}$. Let us now define the observation vector $\mathbf{s}$ for a projected line (conic) in the central catadioptric image as

$$
\mathbf{s}_{k}=\left[\begin{array}{ll}
B_{k 3} & B_{k 4}
\end{array}\right]^{\top}
$$

and the observation vector for $n$ conics as $\mathbf{s}=\left[\mathbf{s}_{1}^{\top}, \ldots, \mathbf{s}_{n}^{\top}\right]^{\top}$. For convenience, in the sequel, we consider only one line and the subscript $k$ will be omitted. Since parameters $B_{i}$ only depend on $\mathbf{n}$, we can write (9) as

$$
\dot{\mathbf{s}}=\mathbf{J}_{\mathrm{sn}} \mathbf{L}_{\mathbf{n}} \boldsymbol{\tau}
$$

where $\mathbf{L}_{\mathbf{n}}$ is the interaction matrix related to the normal vector $\mathbf{n}=\left[n_{x}, n_{y}, n_{z}\right]^{\top}$ to the interpretation plane for line $\mathcal{L}_{i}$ expressed in the mirror frame (such that $\dot{\mathbf{n}}=\mathbf{L}_{\mathbf{n}} \boldsymbol{\tau}$ ), and $\mathbf{J}_{\mathbf{s n}}=\partial \mathbf{s} / \partial \mathbf{n}$.

The interaction matrix related to the observation vector $\mathbf{s}$ is $\mathbf{L}=\mathbf{J}_{\mathrm{sn}} \mathbf{L}_{\mathrm{n}}$. It can be shown that [1], [39]

$$
\dot{\mathbf{n}}=\mathbf{L}_{\mathbf{n}} \boldsymbol{\tau}=\frac{\mathbf{v}^{\top} \mathbf{n}}{h}(\mathbf{u} \times \mathbf{n})-\boldsymbol{\omega} \times \mathbf{n}
$$

where $h=\|\overrightarrow{M \mathcal{P}} \times \mathbf{u}\|$ is the orthogonal distance from $\mathcal{L}_{i}$ to the origin of the mirror frame. According to the previous equation, the interaction between the normal vector and the sensor motion, is thus

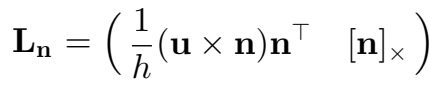

$$
\begin{aligned}
& =\left(\begin{array}{cc}
\frac{1}{h}[\mathbf{u}]_{\times} \mathbf{n n}^{\top} \quad[\mathbf{n}]_{\times}
\end{array}\right) \\
& =\left(\begin{array}{ll}
\mathbf{U}_{h} \mathbf{N} & \mathbf{N}_{\times}
\end{array}\right)
\end{aligned}
$$

where $\mathbf{N}_{\times}=[\mathbf{n}]_{\times}$denotes the antisymmetric matrix associated to the vector $\mathbf{n}, \mathbf{N} .=\mathbf{n n}^{T}$, and $\mathbf{U}_{h}=(1 / h)[\mathbf{u}]_{\times}$. Note that the matrices $\mathbf{N}_{\times}$and $\mathbf{N}$. can be computed using the visual features s (7)

$$
\begin{aligned}
& \mathbf{N}_{\times}=b\left(\begin{array}{ccc}
0 & -1 & B_{4} \\
1 & 0 & -B_{3} \\
-B_{4} & B_{3} & 0
\end{array}\right) \\
& \mathbf{N} .=b^{2}\left(\begin{array}{ccc}
B_{3}^{2} & B_{3} B_{4} & B_{3} \\
B_{3} B_{4} & B_{4}^{2} & B_{4} \\
B_{3} & B_{4} & 1
\end{array}\right) .
\end{aligned}
$$

The Jacobian $\mathbf{J}_{\mathbf{s n}}$ is obtained by computing the partial derivative of (10) with respect to $\mathbf{n}$ and using (7)

$$
\mathbf{J}_{\mathbf{s n}}=\frac{1}{b}\left(\begin{array}{ccc}
1 & 0 & -B_{3} \\
0 & 1 & -B_{4}
\end{array}\right) .
$$

By combining (12) and (14), and according to (11), the interaction matrix $\mathbf{L}$ is

$$
\mathbf{L}=\left(\begin{array}{ll}
\frac{1}{h b} \mathbf{A} & \mathbf{B}
\end{array}\right)
$$

where

$$
\mathbf{A}=\left(\begin{array}{ccc}
u_{y} B_{3} & u_{y} B_{4} & u_{y} \\
-u_{x} B_{3} & -u_{x} B_{4} & -u_{x}
\end{array}\right)
$$

and

$$
\mathbf{B}=\left(\begin{array}{ccc}
B_{3} B_{4} & -1-B_{3}^{2} & B_{4} \\
1+B_{4}^{2} & -B_{3} B_{4} & -B_{3}
\end{array}\right)
$$

In the sequel, we will see how the modeling described in this section can be exploited to design an image-based control law for a 6 DOF holonomic robot and to design a framework to control a nonholonomic mobile robot.

\section{Visual SERVOING OF A 6 DOF Robotic ARM}

\section{A. Control Law}

Consider the vectors $\mathbf{s}=\left[\mathbf{s}_{\mathbf{1}}^{\top}, \mathbf{s}_{\mathbf{2}}^{\top}, \ldots, \mathbf{s}_{\mathbf{n}}^{\top}\right]^{\top}$ and $\mathbf{s}^{*}=$ $\left[\mathbf{s}_{1}{ }^{* \top}, \mathbf{s}_{2}{ }^{* \top}, \ldots, \mathbf{s}_{\mathbf{n}}{ }^{* \top}\right]^{\top}$ where $\mathbf{s}_{\mathbf{i}}$ and $\mathbf{s}_{\mathbf{i}}{ }^{*}$ are $m$-dimensional vectors containing the visual observations at the current and desired configurations of the robotic system. In order to control the movements of a robot from visual features, one defines a task function to be regulated to $\mathbf{0}$ as [41]

$$
\mathbf{e}=\widehat{\mathbf{L}}^{+}\left(\mathbf{s}-\mathbf{s}^{*}\right)
$$

where $\hat{\mathbf{L}}^{+}$is the pseudoinverse of a chosen model of the $(n . m) \times$ 6 interaction matrix $\mathbf{L}$. If the 3-D features corresponding to visual observations are motionless, we get

$$
\dot{\mathrm{s}}=\mathbf{L} \tau \text {. }
$$

A very simple control law can be designed by trying to ensure a decoupled exponential decay of the task function [16], [23]

$$
\boldsymbol{\tau}=-\lambda \mathbf{e}=-\lambda \widehat{\mathbf{L}}^{+}\left(\mathbf{s}-\mathbf{s}^{*}\right) .
$$

In order to compute the control law (20), it is necessary to provide an approximated interaction matrix $\widehat{\mathbf{L}}$. In the cases of projected lines (conics), as can be seen in (16) and (17), only the 3-D parameters $u_{x} / h$ and $u_{y} / h$ have to be introduced in the interaction matrix. As usual, when visual data are used in image-based control, these parameters only act on the translational velocities. The value of $\mathbf{L}$ at the desired position is a typical choice [10] for $\widehat{\mathbf{L}}$. In this case, the 3-D parameters have to be estimated only for the desired position. It is well known that if the interaction matrix is full rank, then the classical (asymptotic) convergence condition holds ( $\mathbf{L} \widehat{\mathbf{L}}^{+}>0$ ). From this condition, it is clear that if the interaction matrix can be perfectly measured, then the convergence is ensured since $\mathbf{L} \widehat{\mathbf{L}}^{+}=\mathbf{I}$. Note that only local (asymptotic) convergence is achieved when the interaction 
matrix of the desired configuration is used in the control law. However, when the interaction matrix cannot be perfectly measured (measurement noise, calibration errors, and errors on 3-D information), then the analysis of the convergence condition is an open problem (in the case of catadioptric camera as well as in the case of conventional camera). Results have been described in [35] for the case where the observation vector $\mathbf{s}$ is defined using the coordinates of projected points and considering only errors on 3-D information.

Note also that at least three lines defining three different interpretation planes are necessary to control the $6 \mathrm{DOF}$ of a robotic arm with the control law (20). If the observation vector $\mathbf{s}$ is defined using only three lines and if the related interaction matrix is full rank, then there is no local minima $(\operatorname{dim}[\operatorname{Ker}(\mathbf{L})]=0)$. However, as when a conventional perspective camera is employed, the same image of three lines can be seen from four different camera poses (four global minima). Indeed, even if three lines impose six constraints on the six motion parameters, the problem of finding the camera pose from three image lines requires solving a nonlinear problem and a high-order polynomial, which may have several solutions (more details can be found in [22] and [15]). A unique pose can be obtained using $n>3$ lines. However, in this case, $\operatorname{dim}[\operatorname{Ker}(\mathbf{L})]=2 n-6$, which implies that local minima may exist. As when using a conventional camera [10], the complexity of the involved symbolic computations seems to make the determination of general results impossible.

Finally, it is important to highlight that the features used to design the control law ( $B_{3}$ and $B_{4}$ ) depend only on the frame where they are defined and not on the camera type. The proposed visual servoing strategy shares thus the same singularity problems as conventional image-based visual servoing with lines. The first potential singularity (decreasing the rank of the interaction matrix) appears when the observation vector $\mathbf{s}$ defines less than three interpretation planes (for instance using only three lines with two of them in the same interpretation plane). A second well known singular configuration appears when the three points of intersection of the three considered lines, belong to a cylinder containing the camera optical center (details about this point can be found in [36]). Using more than three lines generally allows us to avoid such singularities. To our knowledge, there are no supplementary singular configurations.

\section{B. Results}

In this section, we present simulation and experimental results of central catadioptric visual servoing from lines for a $6 \mathrm{DOF}$ robot manipulator.

1) Simulation results with an eye-in-hand system: In this section, we present simulation results of a positioning task of a 6 DOF eye-in-hand robotic system. The positioning task corresponds to an arbitrary motion of translation $\left[\begin{array}{lll}80 & 50 & 20\end{array}\right]^{\top}$ $\mathrm{cm}$ and rotation $\left[\begin{array}{lll}26 & 22 & -45\end{array}\right]^{\top}$ degrees. The value of $\mathbf{L}$ at the desired position has been used. From an initial position, the robot has to reach a desired position expressed as a desired observation vector. Only results involving a sensor composed of a parabolic mirror and an orthographic camera are presented

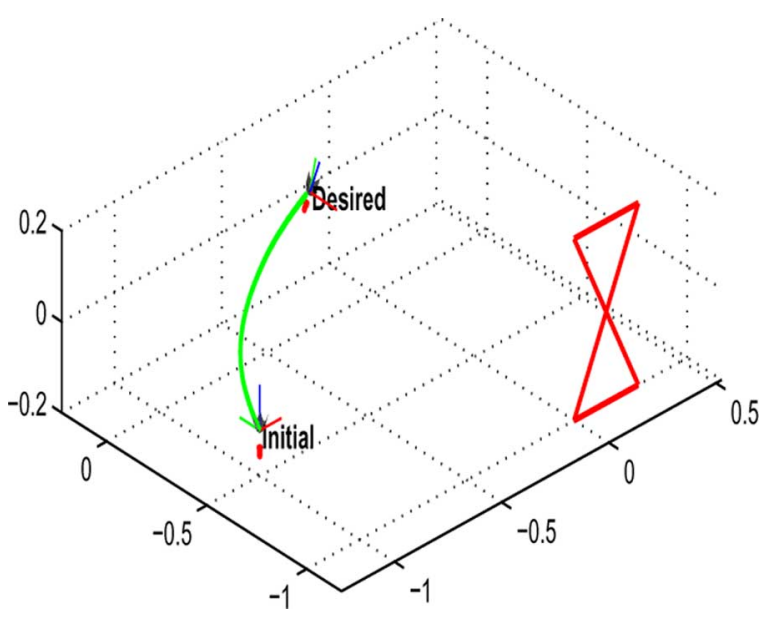

Fig. 3. Line configurations and camera trajectory in 3-D.

here. Similar results can be obtained using sensors composed of a hyperbolic mirror and a conventional camera.

Four lines are used in this simulation and are defined in the world space with the following Plücker coordinates

$$
\left\{\begin{array}{l}
\mathcal{L}_{1}: u_{1}=\left[\begin{array}{lll}
-1 & 0 & 0
\end{array}\right]^{\top}, \quad n_{1}=\left[\begin{array}{lll}
0 & -0.514 & -0.857
\end{array}\right]^{\top} \\
\mathcal{L}_{2}: u_{2}=\left[\begin{array}{lll}
-1 & 0 & 0
\end{array}\right]^{\top}, \quad n_{2}=\left[\begin{array}{lll}
0 & -0.196 & 0.980
\end{array}\right]^{\top} \\
\mathcal{L}_{3}: u_{3}=\left[\begin{array}{lll}
-0.6 & 0 & 0.8
\end{array}\right]^{\top}, \quad n_{3}=\left[\begin{array}{lll}
-0.363 & -0.890 & -0.272
\end{array}\right]^{\top} \\
\mathcal{L}_{4}: u_{3}=\left[\begin{array}{lll}
0.6 & 0 & 0.8
\end{array}\right]^{\top}, \quad n_{3}=\left[\begin{array}{lll}
-0.402 & -0.864 & 0.301
\end{array}\right]^{\top} .
\end{array}\right.
$$

Fig. 3 shows the initial and desired spatial configuration of the lines, the camera, and the estimated trajectory of the camera. To simulate a real setup, image noise has been added to the conic curve which is estimated from noisy data in real situation. The exact conic of the projected 3-D line in the catadioptric image plane is sampled with a step of 10 pixels. An uniformly distributed random noise with a variance of 2 pixels is added to the sampled points in the direction of the normal vector to the exact conic curve. The obtained points are fitted to get the coefficients of the conic curve. Furthermore, the direction vectors of the considered lines with respect to the world frame have been perturbed with errors of maximal amplitude of $5 \%$ (these errors disturb the value of the interaction matrix at the desired configuration). The images corresponding to the initial and desired cameras positions are given in Fig. 4. This figure also shows the trajectories of the conic in the image plane (only the trajectory of one conic is plotted). Camera velocities are given in Fig. 5(a) and (b). As can been seen in Fig. 5(c), the errors between desired and current observation vectors converge toward zero meaning that the positioning task is correctly realized.

2) Experimental results with an eye-to-hand system: The proposed control law has been validated on a 6 DOF eye-tohand system (Fig. 6). In this configuration, the interaction matrix has to take into account the mapping from the camera frame onto the robot control frame [18]. If we denote this mapping by $\left[\mathbf{R}_{e}, \mathbf{t}_{e}\right]$, the eye-to-hand interaction matrix $\mathbf{L}_{e}$ is related to the eye-in-hand one $\mathbf{L}$ by

$$
\mathbf{L}_{e}=\mathbf{L}\left[\begin{array}{cc}
\mathbf{R}_{e} & {\left[\mathbf{t}_{e}\right]_{\times} \mathbf{R}_{e}} \\
\mathbf{0}_{\mathbf{3}} & \mathbf{R}_{e}
\end{array}\right]
$$




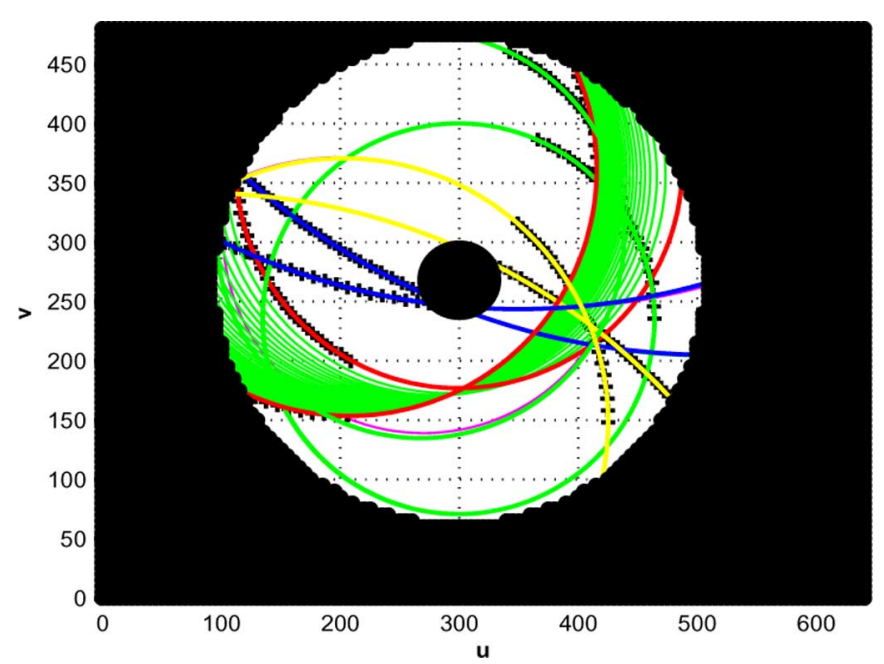

Fig. 4. Trajectories of conics in the image plane.

where $\left[\mathbf{t}_{e}\right]_{\times}$is the skew symmetric matrix associated with translation vector $\mathbf{t}_{e}$. The interaction matrix $\mathbf{L}_{e}$ is used in the control law (20). Since we were not interested in image processing in this paper, the target is composed of white marks (Fig. 6) from which straight lines can be defined [Fig. 7(a)]. The coordinates of these points (the center of gravity of each mark) are extracted and tracked using the Visual Servoing Platform (ViSP) library [30]. The omnidirectional camera used is a parabolic mirror combined with an orthographic lens $(\xi=1)$. Calibration parameters of the camera are: $\alpha_{u}(\phi-\xi)=\alpha_{v}(\phi-\xi)=161$ and $\alpha_{u v}=0$, and the coordinates of the principal point are $\left[\begin{array}{ll}300 & 270\end{array}\right]^{\top}$. From an initial position the robot has to reach a desired position given by a desired 2-D observation vector $\mathbf{s}^{*}$. The image corresponding to the desired and initial configurations are given in Fig. 7(a) and (b), respectively. The corresponding object displacement is composed of a translation $\mathbf{t}=\left[\begin{array}{lll}-10-80 & 60\end{array}\right]^{\top} \mathrm{cm}$ and a rotation (expressed as a rotational vector) $\theta \mathbf{u}=\left[\begin{array}{lll}0 & 0 & 100\end{array}\right]^{\top}$ degrees. Two experiments are presented. In the first one, whose results are depicted in Fig. 8, the intrinsic parameters were taken as mentioned previously. The error between the visual features (desired and current) are plotted on Fig. 8(c) while the camera velocities are plotted on Fig. 8(a) and (b). These results confirm that the positioning task is correctly achieved. The trajectory of the conics in the image are plotted on Fig. 7(b) (for readability's sake, only trajectories of two conics are drawn). In order to check experimentally the robustness with respect to calibration errors, a second experiment has been conducted. The calibration parameters were taken as $\alpha_{u}(\phi-\xi)=180, \alpha_{v}(\phi-\xi)=140$, and the coordinates of the principal point as $\left[\begin{array}{ll}290 & 260\end{array}\right]^{\top}$. The corresponding results are depicted in Fig. 9. It can be noted that the system still converges.

\section{Line Following With a Mobile Robot}

In this sequel, a nonholonomic system is considered with car-like kinematics and the embedded catadioptric system looks upwards from the ground. In the first part of this section, the

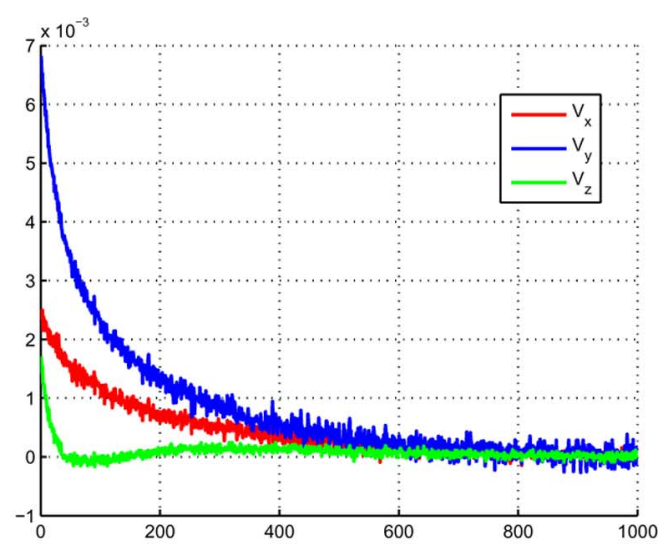

(a)

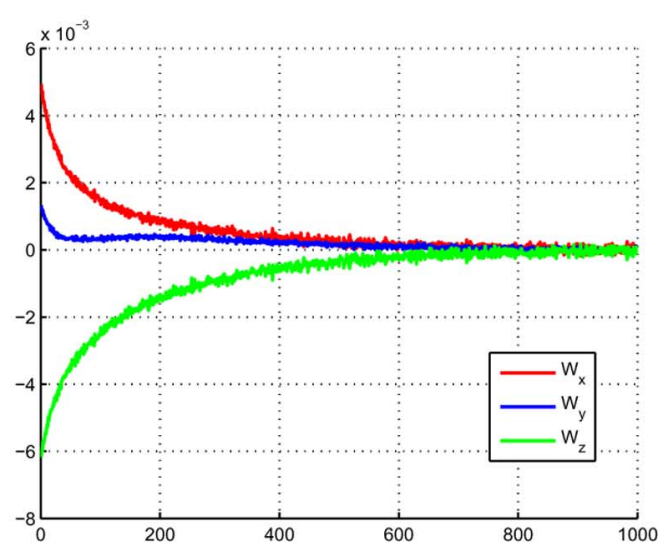

(b)

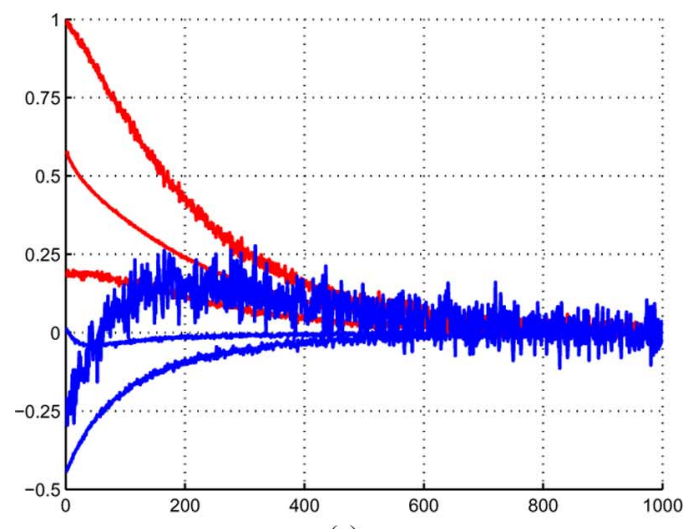

(c)

Fig. 5. (a) Translational velocities (in meter per second). (b) Rotational velocities (in radian per second). (c) Error between desired and current observation vectors $\left(\mathbf{s}-\mathbf{s}^{*}\right)$ versus iteration number.

control objective is presented in the robot workspace and in the catadioptric image space. It is shown that the observation vector $\mathbf{s}=\left[\begin{array}{ll}B_{3} & B_{4}\end{array}\right]^{T}$ (defined in Section II and used to control a manipulator) allows us to design a control scheme based on chained system formalism. In the second part, we present simulations and experimental results.

\section{A. Control Law}

In the sequel, we assume that the camera optical axis is superposed with the rotation axis of the mobile robot. The camera 


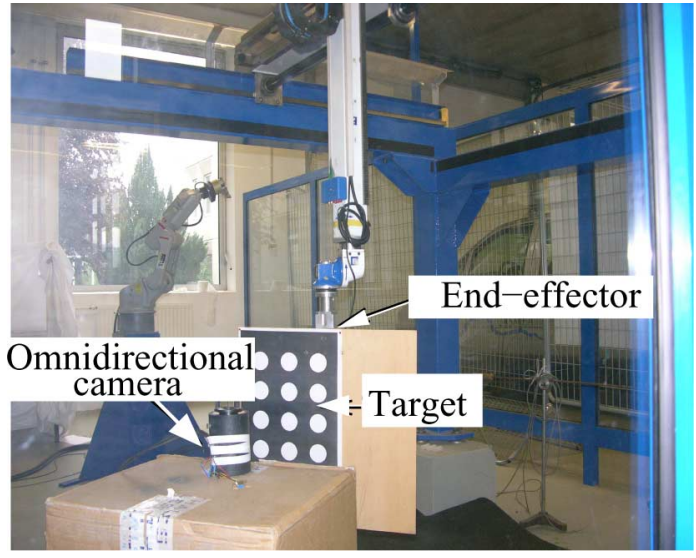

Fig. 6. Experimental setup : eye-to-hand configuration.

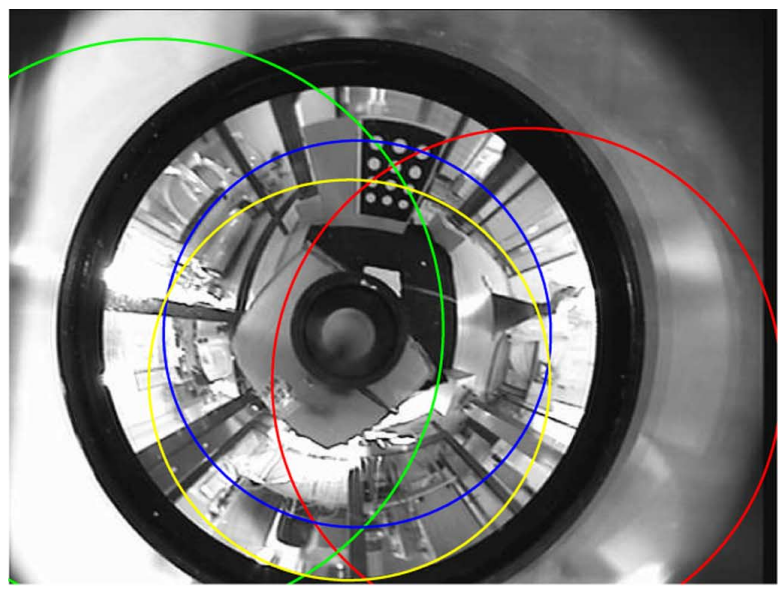

(a)

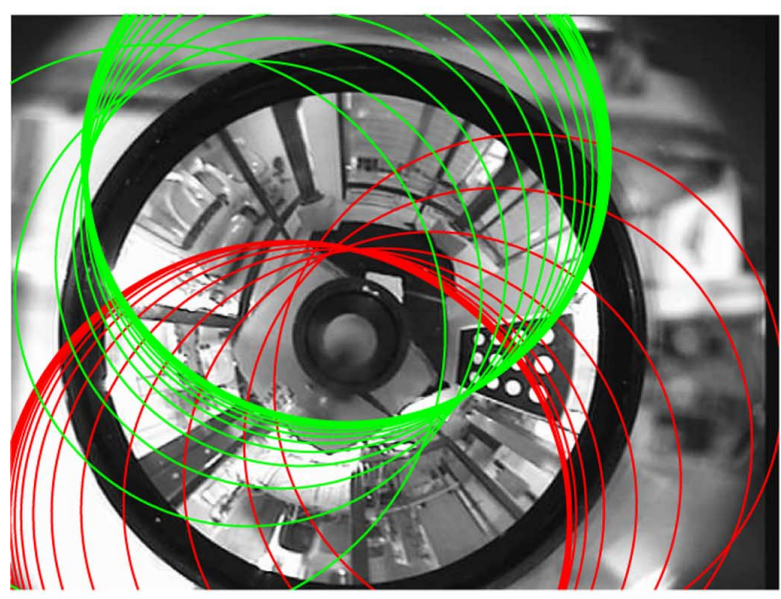

(b)

Fig. 7. 2-D visual servoing from lines. (a) Initial image. (b) Desired image and trajectories of conics (for readability's sake, only trajectories of two conics are drawn).

frame and the mobile robot are subjected to the same kinematic constraints. The kinematic screw is only composed with a linear velocity $v$ along the $X$-axis of the camera frame and an angular velocity $\omega$ about its optical axis.

Consider now a 3-D straight line $\mathcal{L}$ parallel to the $X Y$-plane of the robot control frame $\mathcal{F}_{r}$ and parallel to the $X$-axis of the

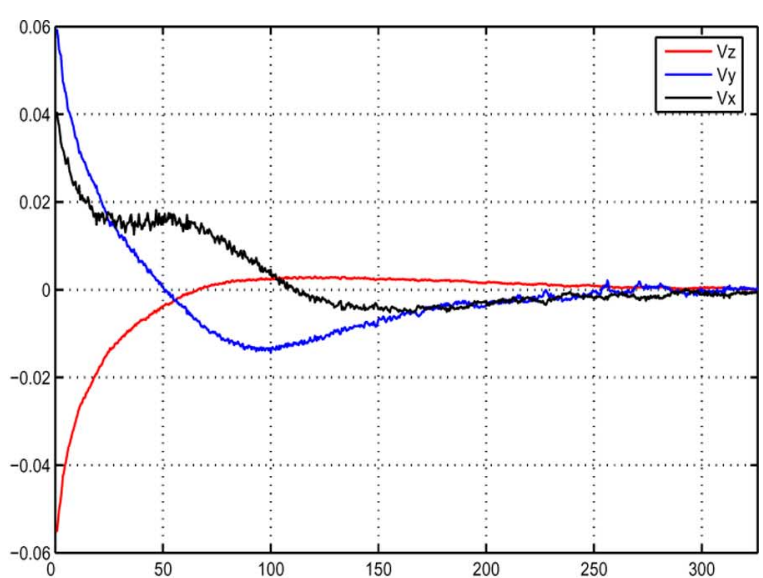

(a)

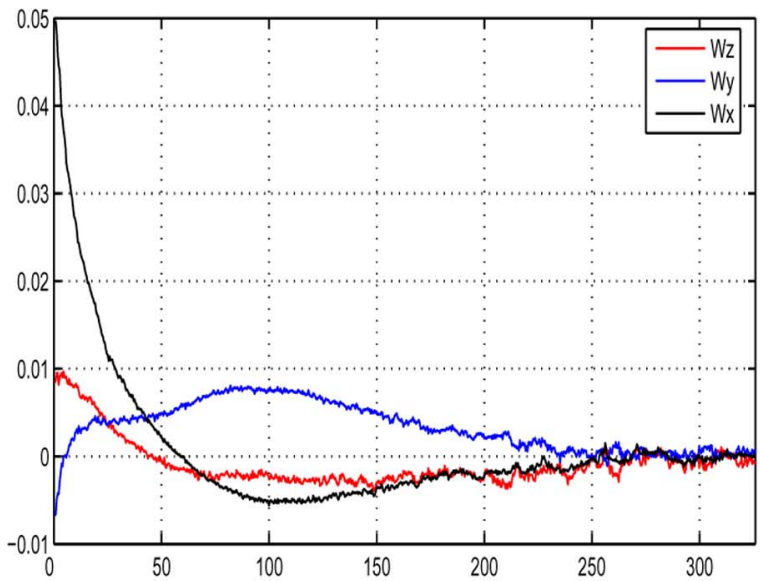

(b)

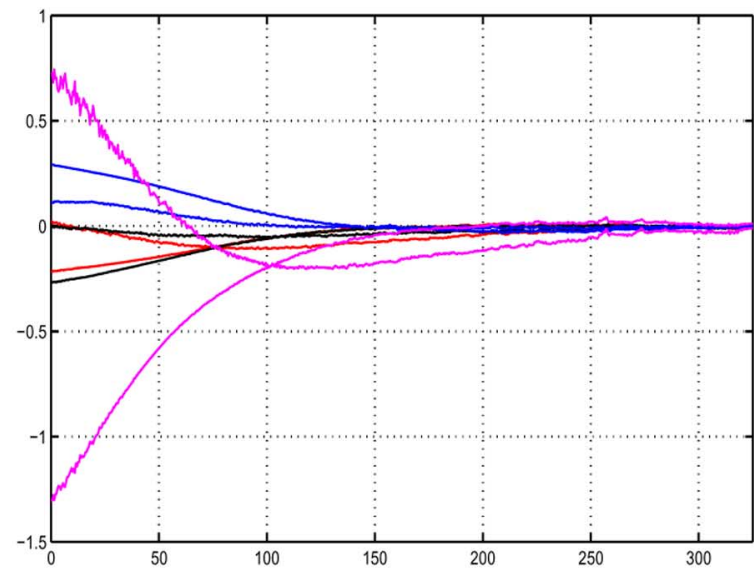

(c)

Fig. 8. Velocity and error vectors. (a) Translational velocities (in meter per second). (b) Rotational velocities (in radian per second). (c) Image error $\mathbf{s}-\mathbf{s}^{*}$ versus iteration number.

world frame. The control objective is to drive the $X$-axis of the control frame parallel to the line while keeping a constant distance to the line (Fig. 10). The state of the mobile robot can be described by the vector $X_{r}=\left[\begin{array}{lll}x & y & \theta\end{array}\right]^{\top}$, where $x$ and $y$ are the coordinates of the camera frame center with respect to the world frame and $\theta$ is the angular deviation with respect to the straight line (Fig. 11). The task is achieved when the lateral deviation 


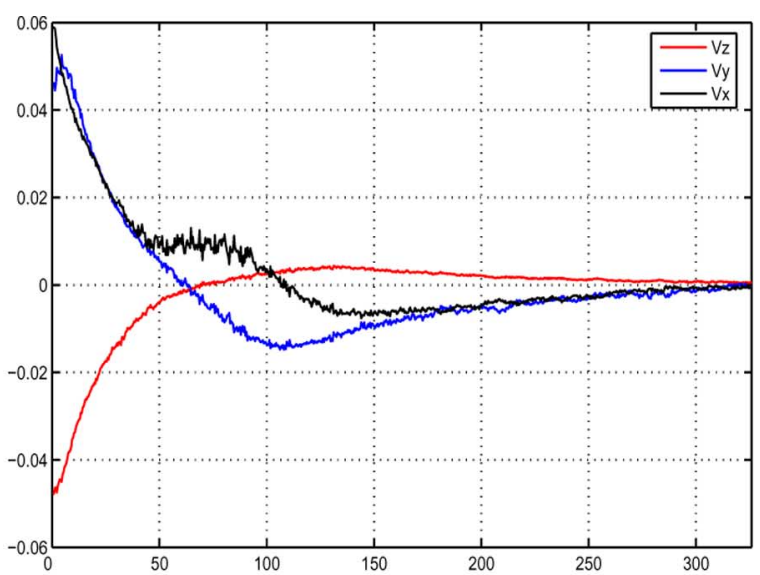

(a)

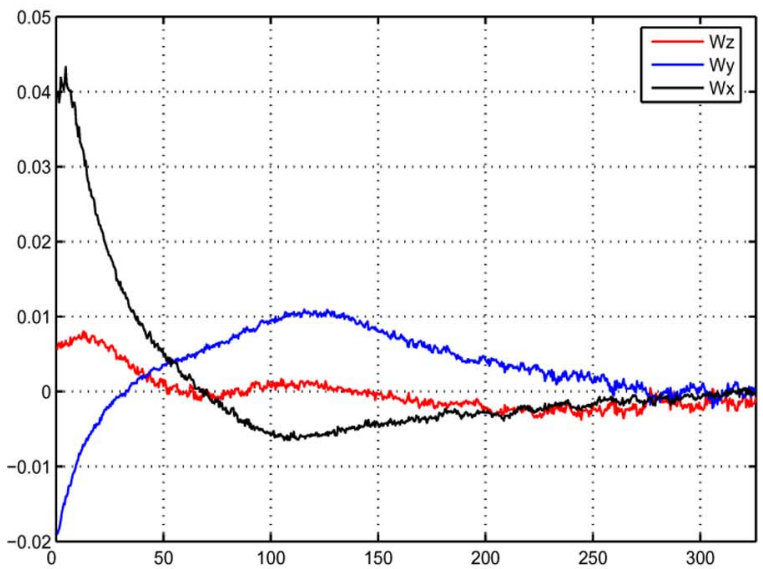

(b)

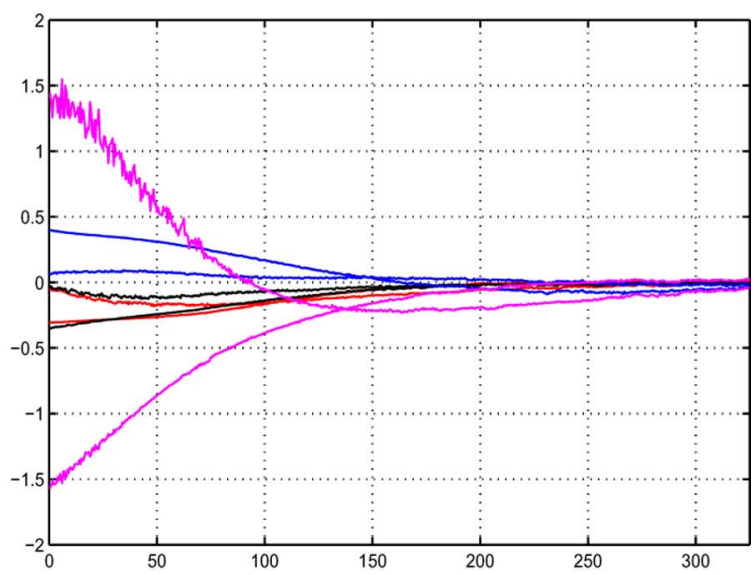

(c)

Fig. 9. Velocity and error vectors. (a) Translational velocities (in meter per second). (b) Rotational velocities (in radian per second). (c) Image error versus iteration number.

$y$ is equal to the desired one $y^{*}$ and the angular deviation $\theta$ is null. Thanks to the properties of chained systems, we are able to decouple the lateral control from the longitudinal deviation if $v \neq 0$. The state vector $X_{r}$ can thus be reduced to $\left[\begin{array}{ll}y & \theta\end{array}\right]^{\top}$. We now describe how to translate the control objective in the catadioptric image space.

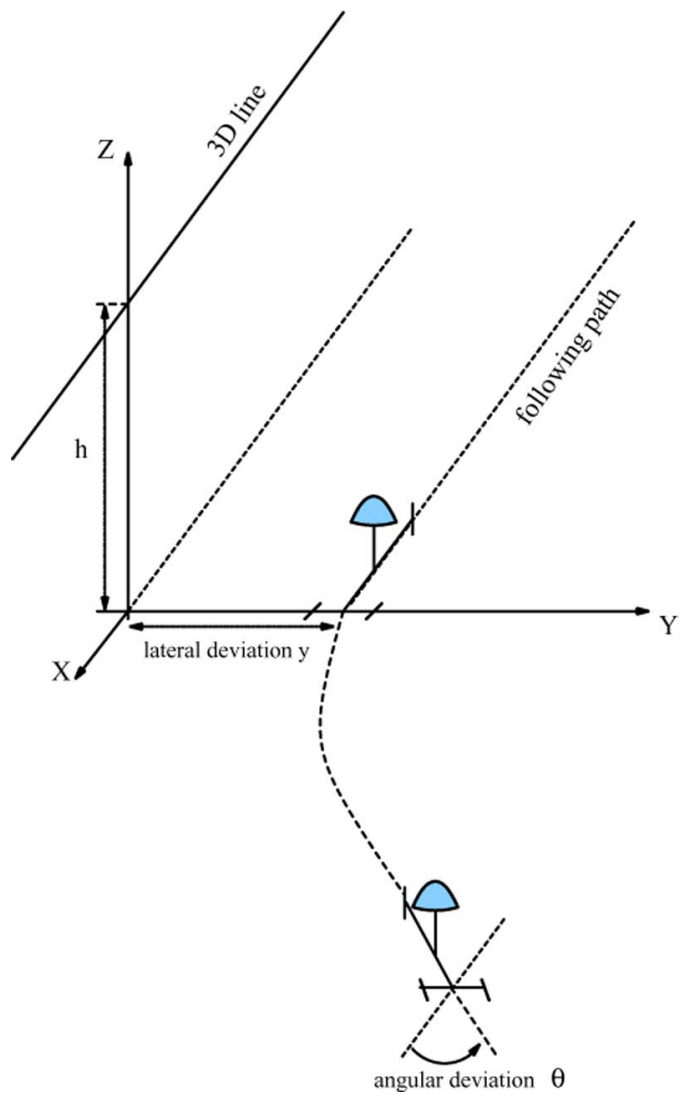

Fig. 10. Task to be achieved.

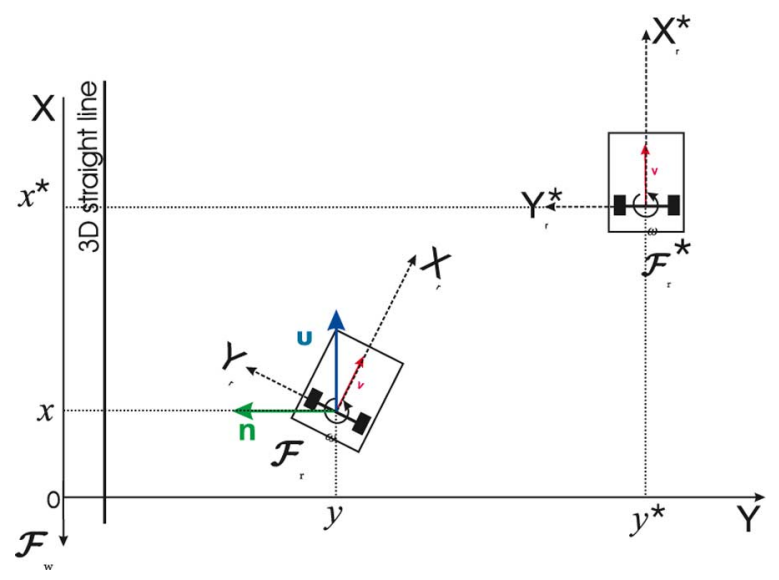

Fig. 11. Modeling the cart-like vehicle.

The projection of a 3-D line $\mathcal{L}$ in the catadioptric image is fully defined by the normal vector $\mathbf{n}$ to the interpretation plane (refer to Section II-B). The direction of $\mathcal{L}$ is given by the unit vector $\mathbf{u}$ of coordinates with respect to the control frame:

$$
\mathbf{u}=\left[\begin{array}{c}
\cos \theta \\
-\sin \theta \\
0
\end{array}\right] \text {. }
$$

The previous relation defines the vector $\mathbf{u}$ as a function of the angular deviation $\theta$ (Fig. 11). It is thus independent of the lateral deviation $y$ of the mobile robot with respect to the line. Since 
$\mathbf{n}=\overrightarrow{M \mathcal{P}} \times \mathbf{u} /\|\overrightarrow{M \mathcal{P}} \times \mathbf{u}\|=\left[n_{x}, n_{y}, n_{z}\right]^{\top}$ is independent of the point $\mathcal{P}$ we choose on the line, $\mathcal{P}$ can be taken as the point $\mathcal{P}=(y \sin \theta, y \cos \theta, h)$ with respect to the control frame $(h$ denotes the height of the line from the ground). The vector normal to the interpretation plane $\mathbf{n}$ is thus given by

$$
\mathbf{n}=\frac{1}{\|\mathbf{n}\|}\left[\begin{array}{c}
-h \sin \theta \\
-h \cos \theta \\
y
\end{array}\right] .
$$

Note that when the $X$-axis of the control frame is parallel to the line $\mathcal{L}$ (i.e when the angular deviation is null) only its last component varies with longitudinal deviation. Similarly, the two first components of $\mathbf{n}$ only depend on the angular deviation. Let us now represent the angular and longitudinal deviation as functions of image features.

Consider the observation vector $\mathbf{s}=\left(B_{3}, B_{4}\right)$ extracted from the projection of line $\mathcal{L}$ in the catadioptric image ( $B_{3}$ and $B_{4}$ have been defined in Section II-B). The observation vector $\mathbf{s}$ fully represents the projection of the line and it is a minimal parameterization. An important remark is that the observation vector $\mathbf{s}$ is the perspective projection of the normal vector $\mathbf{n}$. Remembering that

$$
\left\{\begin{array}{l}
B_{3}=\frac{n_{x}}{n_{z}} \\
B_{4}=\frac{n_{y}}{n_{z}} .
\end{array}\right.
$$

One obtains using (22) and (23)

$$
y=\frac{h}{\sqrt{B_{3}^{2}+B_{4}^{2}}} .
$$

Note that $B_{3}^{2}+B_{4}^{2}$ is null only if the 3-D line lies on the $X Y$ plane of the mirror frame $\mathcal{F}_{m}$. The angular deviation can easily be rewritten as a function of the observation vector by combining (22) and (23)

$$
\theta=\arctan \frac{B_{3}}{B_{4}}
$$

The reduced state vector of the mobile robot $[y \theta]^{\top}$ can thus be expressed directly in the sensor space according to (24) and (25). The control objective is to drive the $X$-axis of the control frame parallel to the line while keeping a constant distance to the line (Fig. 10). The task is achieved when the lateral deviation $y_{e}=y-y^{*}$ and the angular deviation $\theta$ are null. To achieve this control objective, chained systems properties are very interesting. A chained system results from a conversion of a nonlinear model into an almost linear one [37], [40]. As long as the robot longitudinal velocity $v$ is nonzero, the performance of a path tracking algorithm can be measured in terms of settling distance. The cart-like vehicle is supposed to move on a perfectly horizontal ground plane withholding the conditions of pure rolling and nonslipping. The control vector is $\mathbf{u}_{\mathbf{c}}=\left[\begin{array}{ll}v & \omega\end{array}\right]^{\top}$. The state and control vectors are related by the following kinematics equations:

$$
\left\{\begin{array}{l}
\dot{x}=v \cos \theta \\
\dot{y}=v \sin \theta \\
\dot{\theta}=\omega .
\end{array}\right.
$$

Note that the kinematic equations can be translated into the image space using the interaction matrix (15). In order to design the control law as simply as possible, the kinematic equations in Cartesian space will be exploited and (24) and (25) will be used to express the control law in the image space.

Let us now convert the state space model (26) into a chained system with a 3-D state vector $\mathbf{A}_{c}=\left[\begin{array}{lll}a_{1} & a_{2} & a_{3}\end{array}\right]^{\top}$ and a 2D control vector $\mathbf{M}_{c}=\left[\begin{array}{ll}m_{1} & m_{2}\end{array}\right]^{\top}$. The general chained form assigned to systems with three states and two inputs is (refer to [40])

$$
\left\{\begin{array}{l}
\dot{a}_{1}=m_{1} \\
\dot{a}_{2}=a_{3} m_{1} \\
\dot{a}_{3}=m_{2} .
\end{array}\right.
$$

In order to verify that a chained system is almost linear, replace the time derivative by a derivation with respect to the state variable $a_{1}$. Using the notations

$$
\frac{d a_{i}}{d a_{1}}=a_{i}^{\prime} \quad \text { and } \quad m_{3}=\frac{m_{2}}{m_{1}}
$$

the chained form (27) can be rewritten

$$
\left\{\begin{array}{l}
a_{1}^{\prime}=1 \\
a_{2}^{\prime}=a_{3} \\
a_{3}^{\prime}=m_{3} .
\end{array}\right.
$$

The last two equations of system (28) constitute clearly a linear system. Since control law performances are expected to be independent of the longitudinal velocity $v$, the variable $a_{1}$, which drives the evolution of the linear system (28), should be homogeneous to the distance covered by the mobile robot. A natural choice is then

$$
a_{1}=x .
$$

Consequently, variables $a_{2}$ and $a_{3}$ have to be related to $y_{e}$ and $\theta$ in an invertible way. For the sake of simplicity, let us choose

$$
a_{2}=y_{e}
$$

Straightforward computations then show that the nonlinear model (26) can actually be converted into chained forms (27) or (28) from the starting choices (29)-(30). More precisely, we can show successively that $m_{1}=\dot{x}=v \cos \theta, \dot{a_{2}}=v \sin \theta=$ $a_{3} m_{1}$, and therefore

$$
a_{3}=\frac{\dot{a_{2}}}{m_{1}}=\tan \theta .
$$

Consequently, $a_{3}$ is not defined for $\theta=\frac{\pi}{2}[\pi]$. The variable $m_{2}$ can be deduced from (27) and (31):

$$
m_{2}=\frac{\omega}{\cos ^{2} \theta} .
$$


The control scheme can now be completed in a very simple way: since the chained form (28) is linear, we are led to choose the following virtual control law

$$
m_{3}=-K_{d} a_{3}-K_{p} a_{2} \quad\left(K_{p}, K_{d}\right) \in \mathcal{R}^{2}
$$

where $m_{3}=m_{2} / m_{1}=\omega\left(v \cos ^{3} \theta\right)$.

As a matter of fact, inserting (32) in (28), leads to

$$
\frac{d a_{2}^{\prime}}{d a_{1}}+K_{d} a_{2}^{\prime}+K_{p} a_{2}=0 .
$$

If the gains $K_{p}$ and $K_{d}$ are strictly positive then (33) implies that $a_{2}$ (and thus $a_{3}$ ) converge to zero, independently of the longitudinal velocity of the vehicle, as long as $v \neq 0$. Since $a_{2}=y_{e}$ and $a_{3}=\tan \theta$, the same conclusion holds for $y_{e}$ and $\theta$ and one obtains the control law

$$
\omega=-v \cos ^{3} \theta\left(K_{d} \tan \theta+K_{p} y\right) .
$$

Moreover, since the evolution of the error dynamics (33) is driven by $a_{1}=x$, the gains $\left(K_{d}, K_{p}\right)$ impose a settling distance instead of a settling time. Consequently, for a given initial error, the mobile robot trajectory will be identical, whatever the value of $v$ is, and even if $v$ is time-varying. Control law performances are therefore velocity independent. The study of the secondorder differential equation (33) can allows us to fix the gains $\left(K_{d}, K_{p}\right)$ for desired control performances.

According to (24) and (25), the control law (34) can be rewritten as

$$
\omega=-v \cos ^{3}\left(\tan ^{-1} \frac{B_{3}}{B_{4}}\right)\left(K_{d} \frac{B_{3}}{B_{4}}+K_{p} \frac{h}{\sqrt{B_{3}^{2}+B_{4}^{2}}}\right) .
$$

The previous equation presents the control law as a function of the image features $B_{3}, B_{4}$ and the constant parameters $h$. In a real setup, $h$ is estimated and taken as $\widehat{h}=h \cdot \Delta h$. However, the last part of the control law (35) can be written as $K_{p}^{\prime}\left(h /\left(\sqrt{B_{3}^{2}+B_{4}^{2}}\right)\right)$ with $K_{p}^{\prime}=K_{p} \Delta h$, this means that a bad estimation of $h$ acts as a factor on the gain $K_{p}$ and thus modifies the control law performances. From (32) and (33), it is clear that parameter $a_{2}$ converges if the scaled gain $K_{p}^{\prime}$ is strictly positive (which is always true). As a consequence, the same conclusion holds for the lateral and angular deviation. In practice, $\Delta h$ is over-estimated to tune the gains. The control law (35) is valid for $\theta \in]-\pi / 2 \pi / 2[$. However, note that the configurations where $\theta$ is close to $\pi / 2\left(B_{4}=0\right)$ can be detected directly in the image by analyzing the parameters $B_{3}$ and $B_{4}$. A simple strategy to avoid the singularity consists on first zeroing $\theta-\theta^{*}$ using (35), with $\theta^{*}$ chosen such that $\left|\theta-\theta^{*}\right|<\pi / 2$ and then zeroing $\theta$ with (35).

\section{B. Results}

In this section, we present simulation and experimental results for a nonhomonomic mobile robot.

1) Simulation results: In this section, we present simulation results of the central catadioptric vision-based control of a mobile robot using the control law (35). In the first simulation, a paracatadioptric system (a parabolic mirror combined with an

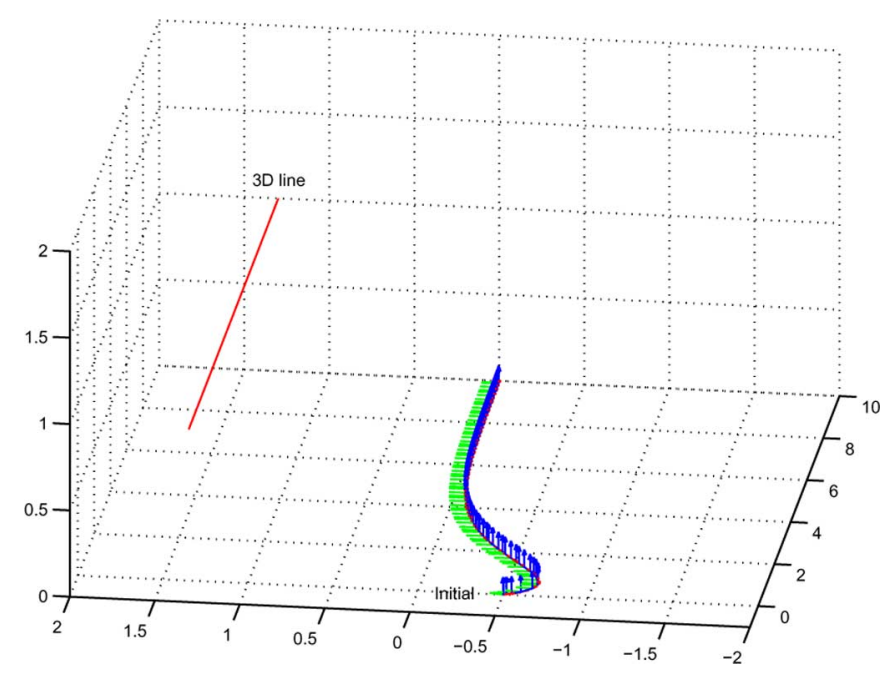

Fig. 12. Line configuration and robot trajectory.

orthographic lens) is used, and in the last one, a hypercatadioptric system (a hyperbolic mirror combined with a perspective lens) is considered. The gains were set to $\left(K_{p}, K_{d}\right)=(1,2)$ and the longitudinal velocity was set to $v=0,1 \mathrm{~ms}^{-1}$ for these simulations. The initial and desired states of the mobile robot with respect to the 3-D line are the same in these simulations. Fig. 12 shows the initial spatial configurations of the line and the camera (or mobile robot). To be close to a real setup, an estimated calibration matrix $\widehat{\mathbf{K}}$ (with an error of $\pm 10 \%$ on the focal length and \pm 5 pixels on the coordinates of the image center) is used. $\widehat{h}$ has been set to $1.2 \mathrm{~m}$ whereas the real value is $1 \mathrm{~m}$, and an image noise has been added when extracting the observation vector $\mathbf{s}$ (maximum amplitude of \pm 5 pixels).

As shown in Figs. 13 and 15, angular and lateral deviations are well regulated to zero in both cases (paracatadioptric and hypercatadioptric cameras). Note also that these deviations are similar in both cases. The projection of the line in the hypercatadioptric image at the initial position of the mobile robot is shown in Fig. 14. It leads to the position corresponding to the desired image given in Fig. 14 when the task is achieved. The trajectory of the projected line in the image is shown in Fig. 14, and it confirms that the task is correctly realized (similar image trajectories are obtained when using a paracatadioptric sensor).

2) Experimental results: In this section, we present experimental results of line following with a mobile robot. The robotic system is composed of a Pioneer 3 mobile robot and of a paracatadioptric camera. The camera is mounted in order to coarsely align the mobile robot rotation axis and the camera optical axis (Fig. 16). The camera has been coarsely calibrated. As in the simulation, the task consists in driving the robot parallel to a 3-D straight line. The projection of the line in the paracatadioptric image at the initial position of the mobile robot is shown in Fig. 17(a). It joins the position corresponding to the desired image given in Fig. 17(b) when the task is achieved. The control law (35) is used to control the mobile robot. As explained previously the height $h$ is over estimated and taken equal to $1.5 \mathrm{~m}$. The settling distance $y^{*}$ has been chosen as $1 \mathrm{~m}$ and the gains $K_{p}$ and $K_{d}$ are 20 and 9 , respectively. Note also that fitting conics 


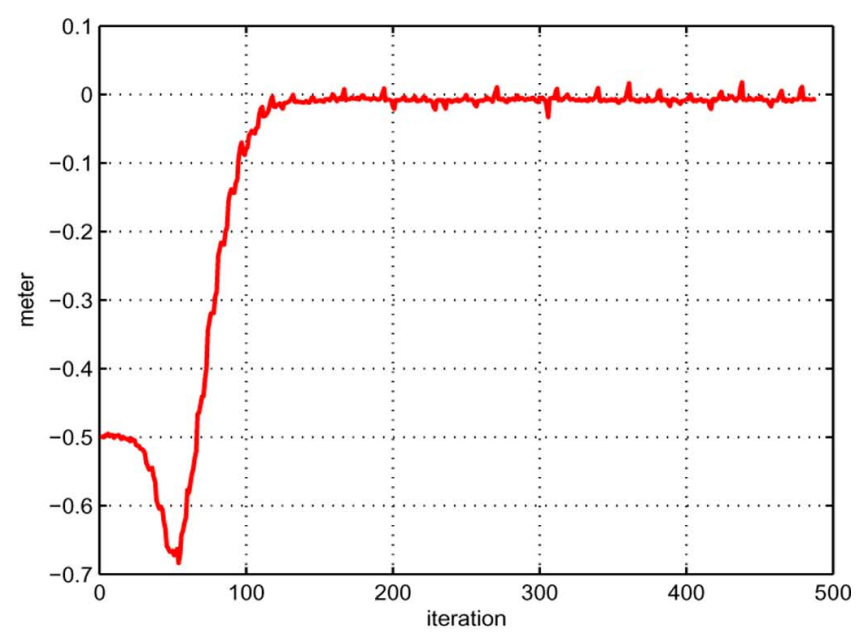

(a)

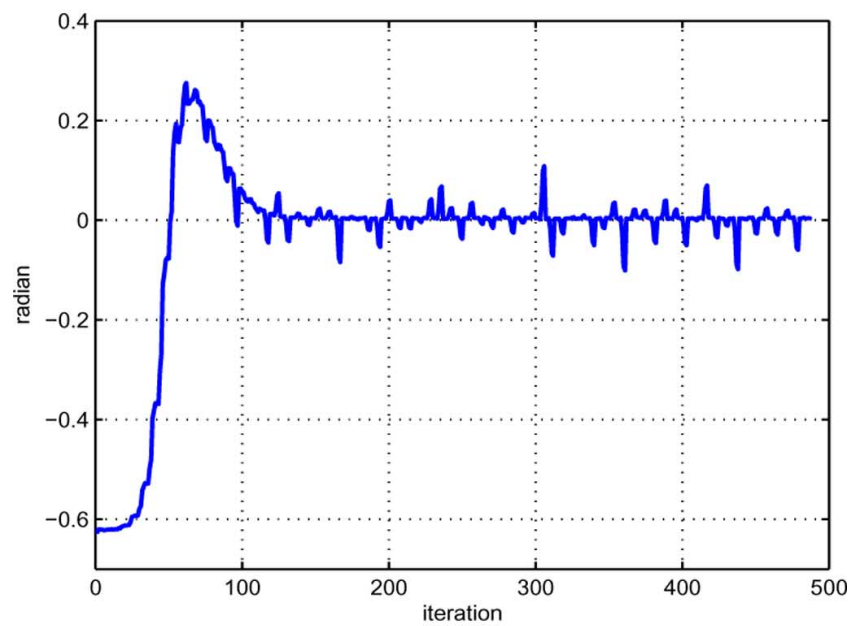

(b)

Fig. 13. Simulation with a paracatadioptric camera. (a) Lateral deviation (in meter). (b) Angular deviation (in radian).

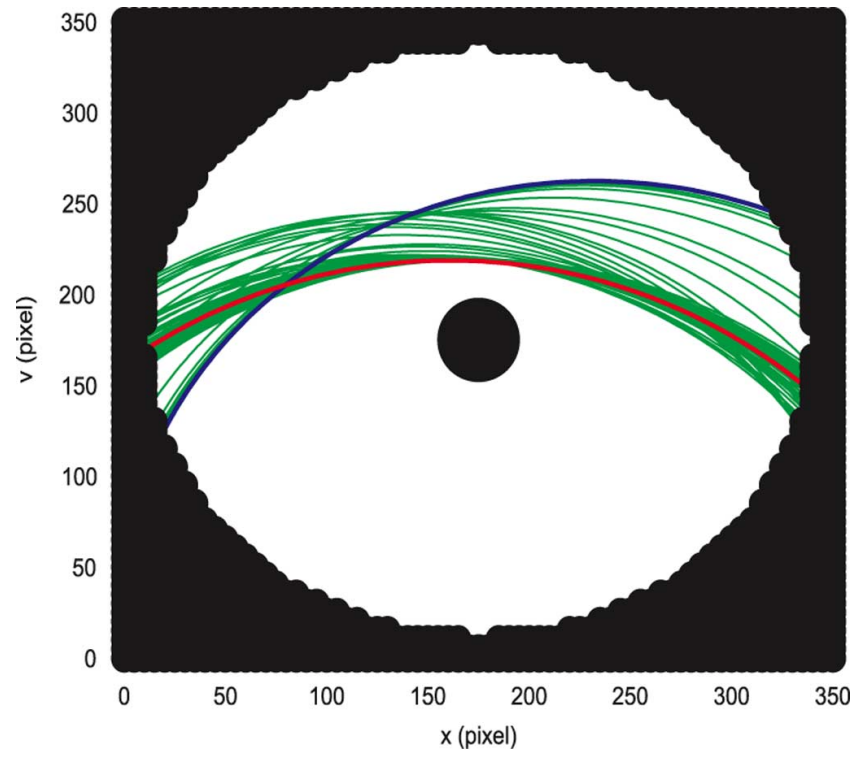

Fig. 14. Trajectories in the image plane of projection of a line with a hypercatadioptric camera.

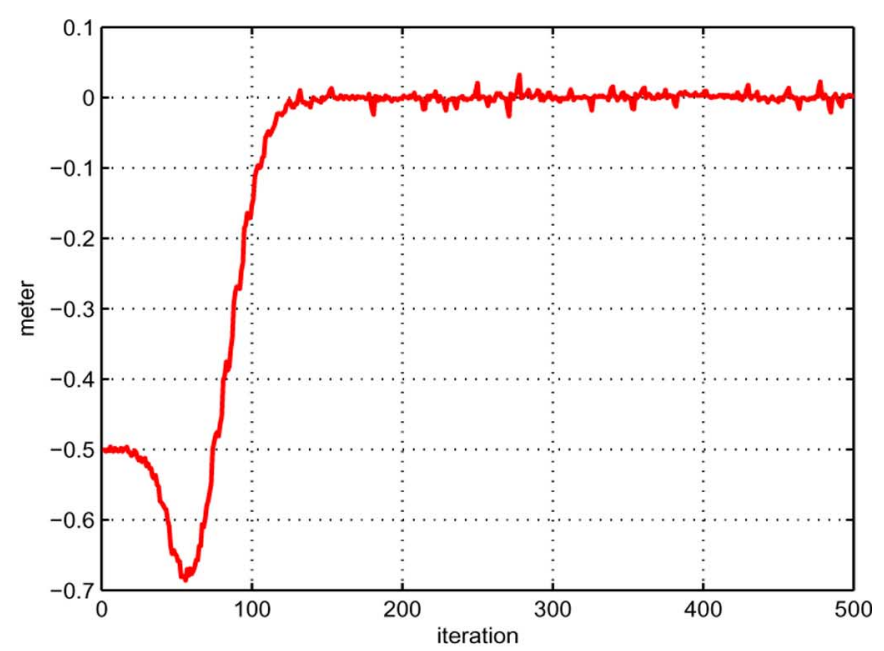

(a)

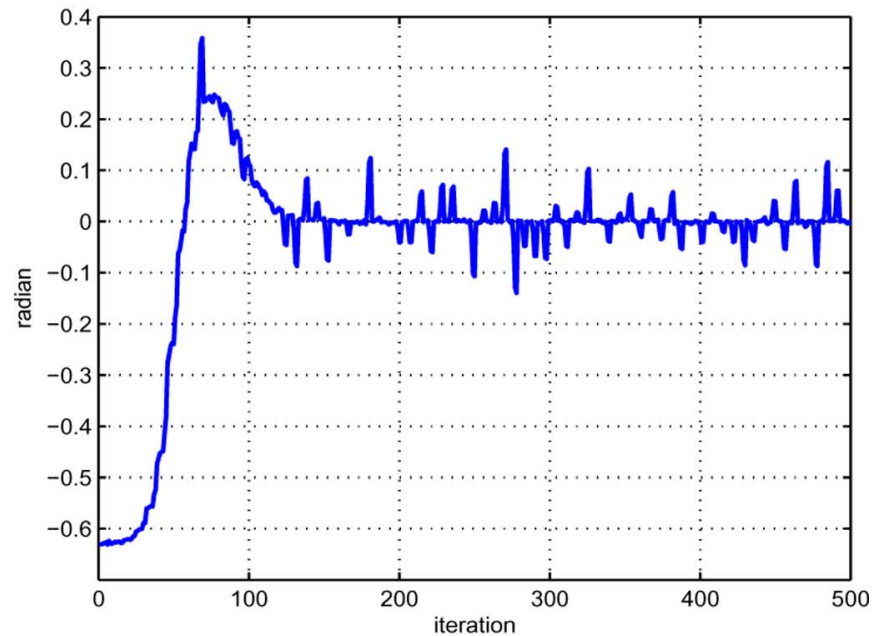

(b)

Fig. 15. Simulation with a hypercatadioptric camera. (a) Lateral deviation (in meter). (b) Angular deviation (in radian).

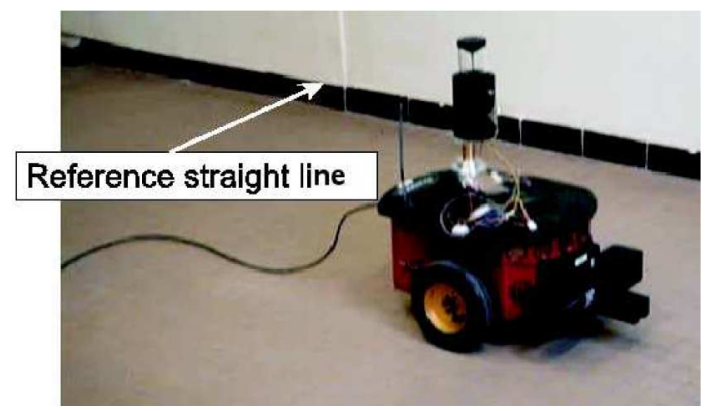

Fig. 16. Mobile robot Pioneer 3 equipped with an omnidirectional camera.

to image measurements is not a simple issue. Such a process is sensitive to noise measurements. Moreover, only a portion of the conic is visible. Fortunately, the projection of 3-D lines onto the image plane of a paracatadioptric camera are circles. In this case, stable and robust algorithms can be used [4], [17]. The circle tracking stage has been implemented by means of the ViSP library [30]. Briefly the tracking algorithm consists 


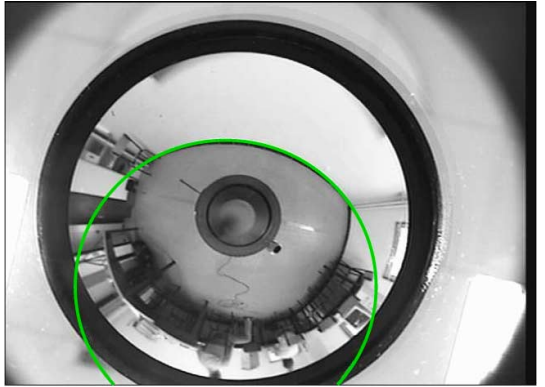

(a)

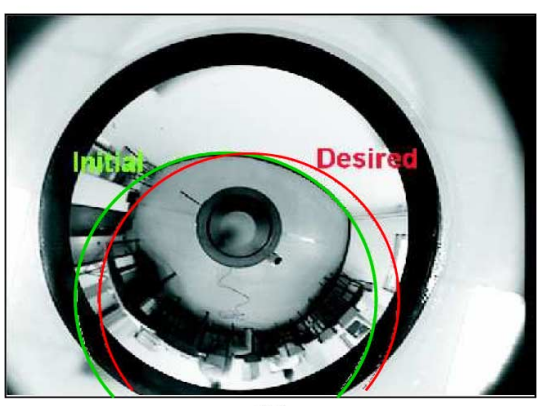

(b)

Fig. 17. (a) Initial image (initial position of the projected straight line). (b) Desired image (initial and desired position of the projected straight line).

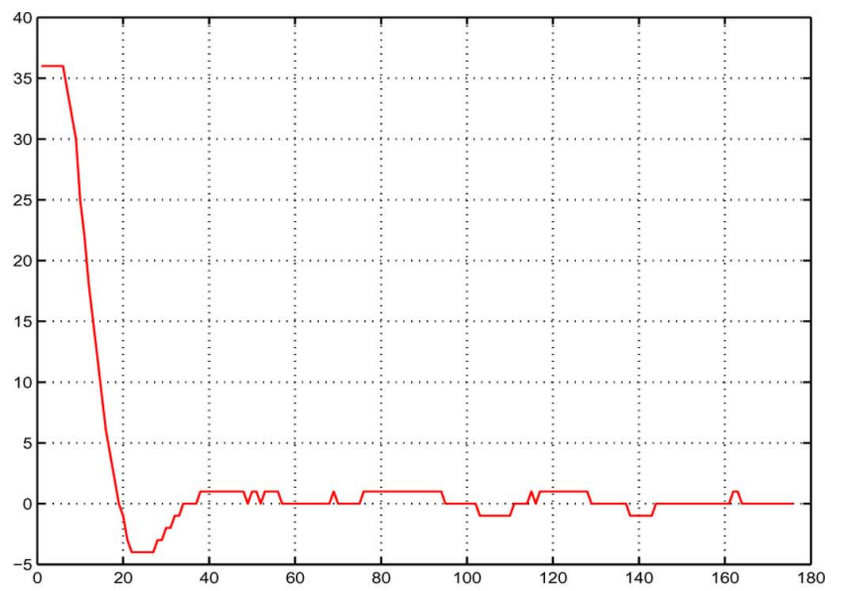

(a)

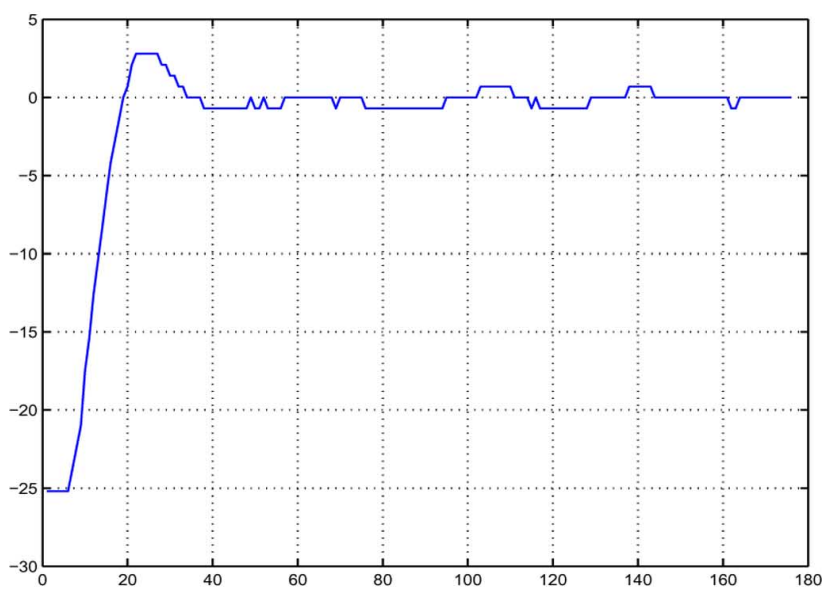

(b)

Fig. 18. (a) Lateral deviation (in centimeter). (b) Angular deviation (in degree). in first sampling the contour points in the first image, second calculating the normal at sample points and seeking the contour on a newly acquired image along the normal using an oriented convolution and third computing the conic parameters with a least square approach [30]. The circles parameters are exploited to compute the control law (35). As shown in Figs. 18(a) and (b), the task is correctly realized since the lateral and angular deviations are well regulated to zero.

\section{CONCLUSION}

We have addressed the problem of controlling a robotic system by incorporating observations from a central catadioptric camera. First an analytical form of the interaction matrix related to the projection of straight lines in catadioptric images has been determined. We have validated the approach with a 6 DOF holonomic robot. We have then detailed the design of a control law suitable for nonholonomic mobile robots based on a chained form of a state vector directly expressed in the image space. The proposed approaches can be used with all central cameras (including conventional ones). In future work, the analytical robustness and stability analysis with respect to the 3-D parameters and calibration errors will be studied.

\section{ACKNOWLEDGMENT}

The authors wish to thank E. Marchand from the Institut de Recherche en Informatique et Systemes Aleatoires (IRISA)/Institut National de Recherche en Informaitique et systemes Aleatoires (INRIA), Rennes, France, who provided them with the software dedicated to circles tracking in omnidirectional images.

\section{REFERENCES}

[1] N. Andreff, B. Espiau, and R. Horaud, "Visual servoing from lines," Int J. Robot. Res., vol. 21, no. 8, pp. 679-700, Aug. 2002.

[2] S. Baker and S. K. Nayar, "A theory of single-viewpoint catadioptric image formation," Int. J. Comput. Vis., vol. 35, no. 2, pp. 1-22, Nov. 1999.

[3] J. Barreto and H. Araujo, "Geometric properties of central catadioptric line images," in Proc. 7th Eur. Conf. Comput. Vis. (ECCV 2002), Copenhagen, Denmark, May, pp. 237-251.

[4] J. Barreto and H. Araujo, "Direct least square fitting of paracatadioptric line images," in Proc. OMNIVIS 2003 Workshop Omnidirectional Vis. Camera Netw., Madison, WI, Jun., pp. 78-83.

[5] J. P. Barreto, F. Martin, and R. Horaud, "Visual servoing/tracking using central catadioptric images," in Proc. 2002 ISER 8th Int. Symp. Exp. Robot., Mumbai, India, Jul., pp. 863-869.

[6] S. Benhimane and E. Malis, "Vision-based control with respect to planar and non-planar objects using a zooming camera," in Proc. IEEE Int. Conf. Adv. Robot., Jul. 2003, pp. 863-869.

[7] R. Benosman and S. Kang, Panoramic Vision. New York: SpringerVerlag, 2000.

[8] P. Blaer and P. K. Allen, "Topological mobile robot localization using fast vision techniques," in Proc. IEEE Int. Conf. Robot. Autom., Washington, May 2002, vol. 1, pp. 1031-1036.

[9] D. Burshka, J. Geiman, and G. Hager, "Optimal landmark configuration for vision based control of mobile robot," in Proc. IEEE Int. Conf. Robot. Autom., Tapei, Taiwan, Sep. 2003, vol. 3, pp. 3917-3922.

[10] F. Chaumette, "Potential problems of stability and convergence in imagebased and position-based visual servoing," in The Confluence of Vision and Control (LNCIS Series 237), D. Kriegman, G. Hager, and A. S. Morse, Eds. New York: Springer-Verlag, 1998, pp. 66-78. 
[11] F. Chaumette, "Image moments: A general and useful set of features for visual servoing," IEEE Trans. Robot., vol. 20, no. 4, pp. 713-723, Aug. 2004.

[12] J. Chen, W. E. Dixon, D. M. Dawson, and M. McIntyre, "Homographybased visual servo tracking control of a wheeled mobile robot," IEEE Trans. Robot., vol. 22, no. 2, pp. 407-415, Apr. 2006.

[13] G. Chesi, K. Hashimoto, D. Prattichizzo, and A. Vicino, "Keeping features in the field of view in eye-in-hand visual servoing: A switching approach," IEEE Trans. Robot., vol. 20, no. 5, pp. 908-913, Oct. 2004.

[14] N. J. Cowan, J. D. Weingarten, and D. E. Koditschek, "Visual servoing via navigation functions," IEEE Trans. Robot. Autom., vol. 18, no. 4, pp. 521-533, Aug. 2002.

[15] M. Dhome, M. Richetin, J. T. Lapreste, and G. Rives, "Determination of the attitude of 3D objects from a single perpective view," IEEE Trans. Pattern Anal. Mach. Intell., vol. 11, no. 12, pp. 1265-1278, Dec. 1989.

[16] B. Espiau, F. Chaumette, and P. Rives, "A new approach to visual servoing in robotics," IEEE Trans. Robot. Autom., vol. 8, no. 3, pp. 313-326, Jun. 1992.

[17] A. Fitzgibbon, M. Pilu, and R. B. Fisher, "Direct least-squares fitting of ellipses," IEEE Trans. Pattern Anal. Mach. Intell., vol. 21, no. 5, pp. 476480, May 1999.

[18] G. Flandin, F. Chaumette, and E. Marchand, "Eye-in-hand/eye-to-hand cooperation for visual servoing," in Proc. IEEE Int. Conf. Robot. Autom. (ICRA 2000), San Francisco, Apr., vol. 3, pp. 2741-2746.

[19] C. Geyer and K. Daniilidis, "A unifying theory for central panoramic systems and practical implications," in Eur. Conf. Comput. Vis., Dublin, Ireland, May 2000, vol. 29, pp. 159-179.

[20] H. Hadj-Abdelkader, Y. Mezouar, N. Andreff, and P. Martinet, "Imagebased control of mobile robot with central catadioptric cameras," in Proc. IEEE Int. Conf. Robot. Autom. (ICRA 2005), Barcelonna, Spain, Apr., pp. 3522-3527.

[21] H. Hadj Abdelkader, Y. Mezouar, and P. Martinet, "Path planning for image based control with omnidirectional cameras," in Proc. 45th IEEE Conf. Decision Control (CDC 2006), San Diego, CA, Dec., pp. 17641769.

[22] R. Horaud, "New methods for matching 3D objects with single perpective view," IEEE Trans. Pattern Anal. Mach. Intell., vol. 9, no. 3, pp. 401-412, May 1987.

[23] S. Hutchinson, G. D. Hager, and P. I. Corke, "A tutorial on visual servo control," IEEE Trans. Robot. Autom., vol. 12, no. 5, pp. 651-670, Oct 1996.

[24] J. Kosecka, "Visually guided navigation," in Proc. 4th Int. Symp. Intell. Robot. Syst. (SIRS 1996), Lisbon, Portugal, Jul., pp. 77-95.

[25] Luca, A. De, G. Oriolo, and C. Samson, "Feedback control of a nonholonomic car-like robot," in Robot Motion Planning and Control (Lecture Notes in Control and Information Sciences), vol. 229, J. P. Laumond, Ed. New York: Springer-Verlag, 1998, pp. 171-253 (ISBN 3-540-76219-1).

[26] Y. Ma, J. Kosecka, and S. S. Sastry, "Vision guided navigation for a nonholonomic mobile robot," IEEE Trans. Robot. Autom., vol. 15, no. 3, pp. 521-537, Jun. 1999.

[27] R. Mahony and T. Hamel, "Visual servoing usinf linear features for underactuated rigid body dynamics," in Intell. Robot. Symp. (IROS 2001), May, vol. 2, pp. 1153-1158.

[28] E. Malis, J. Borrelly, and P. Rives, "Intrinsics-free visual servoing with respect to straight lines," in IEEE/RSJ Int. Conf. Intell. Robots Syst., Lausanne, Switzerland, Oct. 2002, vol. 1, pp. 384-389.

[29] E. Malis, F. Chaumette, and S. Boudet, "2 1/2 D visual servoing/," IEEE Trans. Robot. Autom., vol. 15, no. 2, pp. 238-250, Apr. 1999.

[30] E. Marchand, F. Spindler, and F. Chaumette, "ViSP for visual servoing: A generic software platform with a wide class of robot control skills," IEEE Robot. Autom. Mag., vol. 12, no. 4, pp. 40-52, Dec. 2005.

[31] G. L. Mariottini, G. Oriolo, and D. Prattichizzo, "Image-based visual servoing for nonholonomic mobile robots with central catadioptric camera using epipolar geometry," IEEE Trans. Robot., vol. 23, no. 1, pp. 87-100, Feb. 2007.

[32] Y. Mezouar and F. Chaumette, "Path planning for robust image-based control," IEEE Trans. Robot. Autom., vol. 18, no. 4, pp. 534-549, Aug. 2002.

[33] Y. Mezouar and F. Chaumette, "Avoiding self-occlusions and preserving visibility by path planning in the image," Robot. Autom. Syst., vol. 41, no. 2, pp. 77-87, Nov. 2002.

[34] Y. Mezouar, H. Haj Abdelkader, P. Martinet, and F. Chaumette, "Central catadioptric visual servoing from 3D straight lines," in IEEE/RSJ Int. Conf. Intell. Robots Syst., IROS 2004, Sendai, Japan, Sep. vol. 1, pp. 343349.
[35] Y. Mezouar and E. Malis, "Robustness of central catadioptric image-based visual servoing to uncertainties on 3D parameters," in IEEE/RSJ Int. Conf. Intell. Robots Syst. (IROS 2004), Sendai, Japan, Sep., pp. 1389-1394.

[36] H. Michel and P. Rives, "Singularities in the determination of the situation of a robot effector from the perspective view of 3 points," INRIA, Tech. Rep. 1850, Feb. 1993.

[37] M. R. Murray and S. S. Sastry, "Nonholonomic motion planning: Steering using sinusoids," IEEE Trans. Autom. Control, vol. 38, no. 5, pp. 700-716, 1993.

[38] A. Paulino and H. Araujo, "Multiple robots in geometric formation: Control structure and sensing," in Proc. Int. Symp. Intell. Robot. Syst., Jul. 2000, pp. 103-112.

[39] P. Rives and B. Espiau, "Closed-loop recursive estimation of 3D features for a mobile vision system," in Proc. IEEE Int. Conf. Robot. Autom. (ICRA 1987), Raleigh, NC, Mar., vol. 4, pp. 1436-1443.

[40] C. Samson, "Control of chained system. application to path following and time-varying stabilization of mobile robot," IEEE Trans. Autom. Control, vol. 40, no. 1, pp. 64-77, Jan. 1995.

[41] C. Samson, B. Espiau, and M. Le Borgne, Robot Control: The Task Function Approach. Oxford, U.K.: Oxford Univ. Press, 1991.

[42] B. Thuilot, P. Martinet, L. Cordesses, and J. Gallice, "Position-based visual servoing: keeping the object in the field of vision," in Proc. IEEE Int. Conf. Robot. Autom., Washington, DC, May 2002, vol. 2, pp. 1624-1629.

[43] D. Tsakiris, P. Rives, and C. Samson, "Extending visual servoing techniques to nonholonomic mobile robots," in The Confluence of Vision and Control (Lecture Notes in Control and Information Science), vol. 237, G. Hager, D. Kriegman, and A. Morse, Eds. New York: Springer-Verlag, 1998, pp. 106-117.

[44] K. Usher, P. Ridly, and P. Corke, "Visual servoing of a car-like vehicleAn application of omnidirectional vision," in Proc. Aust. Conf. Robot. Autom., Auckland, Nov. 2002, pp. 37-42.

[45] R. Vidal, O. Shakernia, and S. Sastry, "Formation control of nonholonomic mobile robots with omnidirectional visual servoing and motion segmentation," in Proc. IEEE Int. Conf. Robot. Autom., Taipei, Taiwan, Sep. 2003, pp. 584-589.

[46] N. Winter, J. Gaspar, G. Lacey, and J. Santos-Victor, "Omnidirectional vision for robot navigation," in Proc. IEEE Workshop Omnidirectional Vis. (OMNIVIS), Jun. 2000, pp. 21-28.

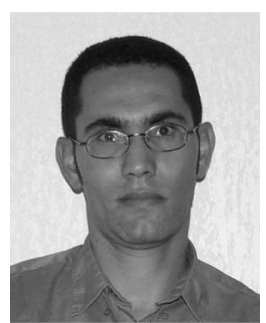

Hicham Hadj-Abdelkader was born in Algeria, in 1977. He received the Ph.D. degree in electronics and systems from the Blaise Pascal University, Aubiere, France, in 2006

He is currently a Postdoctoral Fellow at the Institut National de Recherche en Informatique et Automatique (INRIA), Sophia Antipolis, France. His current research interests include robotics, computer vision, and vision-based control.

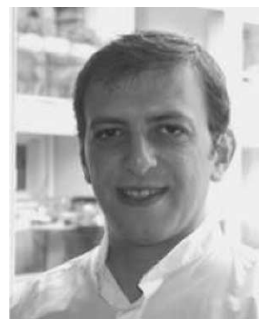

Youcef Mezouar was born in Paris, France, in 1973 He received the Ph.D. degree in computer science from the University of Rennes 1, Rennes, France, in 2001.

He was a Postdoctoral Associate in the Robotics Laboratory of the Computer Science Department, Columbia University, New York, NY. Since 2002, he has been with the Robotics and Vision Group, Laboratoire des Sciences et Materiaux pour 1'Electronique et d'Automatique (LASMEA)-Centre National de la Recherche Scientifique (CNRS), Aubiere, France. His current research interests include robotics, computer vision, vision-based control, and mobile robots navigation. 


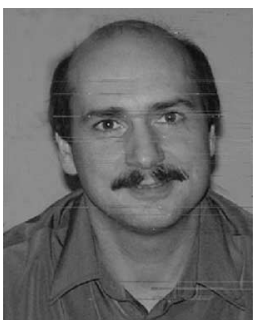

Philippe Martinet received the Graduate degree in electronics from the Center Universitaire Scientifique et Technique (CUST), Clermont-Ferrand, France, in 1985, and the Ph.D. degree in electronics science from the Blaise Pascal University, Aubiere, France, in 1987.

From 1990 to 2000, he was an Assistant Professor in the Department of Electrical Engineering, CUST. Since 2000, he has been a Professor at the Institut Français de Mécanique Avancée (IFMA), ClermontFerrand. From 2001 to 2006 , he was with the Groupe Automatique Vision et Robotique (GRAVIR) as a Leader. During 2006-2007, he was a Visiting Professor in the Intelligent Systems Research Center (ISRC), Sungkyunkwan University, Seoul, Korea. He is currently with the Robotics and Vision Group, LAboratoire des Sciences et Materiaux pour l'Electronique et d'Automatique (LASMEA)-Center National de la Recherche Scientifique (CNRS), Aubiere. He is also leading the RObotic and Autonomous ComplEx System (ROSACE) Team. His current research interests include visual servoing, multisensor-based control, force-vision coupling, autonoumous guided vehicle control, enhanced mobility (sliding and slipping), platoon, multirobot, modeling identification and control of complex machines kinematic identification, dynamic identification modeling and control, vision-based control of parallel robot. He is the author or coauthor of more than 160 references.

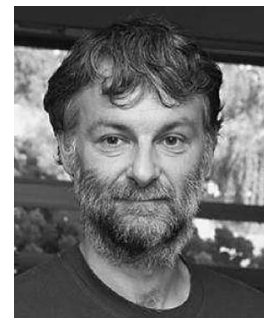

François Chaumette (M'98) received the Graduate degree in automatic control from École Nationale Supérieure de Mécanique, Nantes, France, in 1987, and the Ph.D. degree in computer science from the University of Rennes, Rennes, France, in 1990.

Since 1990, he has been with the Institut de Recherche en Informatique et Systemes Aleatoires (IRISA), Rennes where he is currently the "Directeur de Recherches" INRIA and the Head of the Lagadic Group (http://www.irisa.fr/lagadic). His current research interests include robotics and computer vision, with special emphasis on visual servoing, and active perception. He is the author or coauthor of more than 150 published papers in the related areas.

Dr. Chaumette was an Associate Editor of the IEEE TRANSACTIONS ON RoвOTICS from 2001 to 2005. He was a member of various program committees of national and international conferences in the field of robotics and computer vision. He was the recipient of the "best French thesis in automatic control" Award in 1991. With Ezio Malis, he was also the recipient of the 2002 King-Sun Fu Memorial Best Paper Award in IEEE Transactions ON ROBOTICS AND AUTOMATION. 